\title{
Combinatorial duality for Poincaré series, polytopes and invariants of plumbed 3-manifolds
}

\author{
Tamás László ${ }^{1,2}$ • János Nagy ${ }^{3}$. András Némethi ${ }^{1,2,4}$ \\ Published online: 27 February 2019 \\ (C) The Author(s) 2019
}

\begin{abstract}
Assume that the link of a complex normal surface singularity is a rational homology sphere. Then its Seiberg-Witten invariant can be computed as the 'periodic constant' of the topological multivariable Poincare series (zeta function). This involves a complicated regularization procedure (via quasipolynomials measuring the asymptotic behaviour of the coefficients). We show that the (a Gorenstein type) symmetry of the zeta function combined with Ehrhart-Macdonald-Stanley reciprocity (of Ehrhart theory of polytopes) provide a simple expression for the periodic cosntant. Using these dualities we also find a multivariable polynomial generalization of the Seiberg-Witten invariant, and we compute it in terms of lattice points of certain polytopes. All these invariants are also determined via lattice point counting, in this way we establish a completely general topological analogue of formulae of Khovanskii and Morales valid for the geometric genus of singularities with non-degenerate Newton principal part.
\end{abstract}

Keywords Normal surface singularities · Links of singularities · Plumbing graphs · Rational homology spheres · Seiberg-Witten invariant · Poincaré series · Quasipolynomials · Surgery formula · Periodic constant · Ehrhart polynomials · Ehrhart-Macdonald-Stanley reciprocity law · Gorenstein duality

$凶$ András Némethi

nemethi.andras@renyi.mta.hu

Tamás László

laszlo.tamas@renyi.mta.hu

János Nagy

nagy_janos@phd.ceu.edu

1 Present Address: Alfréd Rényi Institute of Mathematics, Hungarian Academy of Sciences, Reáltanoda utca 13-15, 1053 Budapest, Hungary

2 BCAM - Basque Center for Applied Math, Mazarredo 14, 48009 Bilbao, Basque Country, Spain

3 Department of Mathematics, Central European University, Budapest, Hungary

4 Department of Geometry, ELTE - University of Budapest, Budapest, Hungary

Birkhäuser 
Mathematics Subject Classification Primary 32S05 - 32S25 - 32S50 - 57M27;

Secondary $14 \mathrm{Bxx} \cdot 14 \mathrm{~J} 80 \cdot 57 \mathrm{R} 57$

\section{Contents}

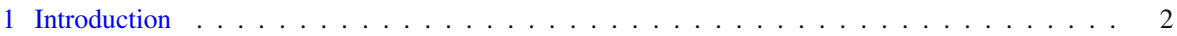

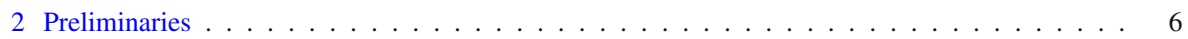

3 Ehrhart-Macdonald-Stanley duality for rational functions . . . . . . . . . . . . . . . . . 10

4 Duality for multivariable Poincaré series . . . . . . . . . . . . . . . . . . . . . 15

5 The 'polynomial part' of the series $\mathcal{Z}(\mathbf{t}) \ldots \ldots \ldots \ldots \ldots \ldots \ldots$

6 Topological polytopes, counting lattice points and Seiberg-Witten invariants . . . . . . . . . . . 21

References................................ 30

\section{Introduction}

\section{1}

In order to study the topological invariants of links of complex normal surface singularities one usually uses the dual resolution graph of the singularity. Indeed, such links appear as plumbed 3-manifolds associated with these graphs, and the combinatorics of the graph is an ideal source to codify the topological invariants, even if they were originally defined by completely different methods of algebraic or differential topology. E.g., in order to determine the Seiberg-Witten invariants of a rational homology sphere link, a possible procedure is the following. Firstly, one defines from the graph a multivariable rational function, the so-called 'zeta function' $\mathcal{Z}(\mathbf{t})$ (it is also called the 'multivariable topological Poincaré series', as the topological analogue of a 'multivariable analytical Poincaré series' introduced by Campillo, Delgado and Gusein-Zade [8,9]). This has a natural decomposition $\mathcal{Z}=\sum_{h \in H} \mathcal{Z}_{h}$, where $H$ is the first homology of the link $M$ (and it indexes also $\operatorname{Spin}^{c}(M)$ ). Then one shows that a summation associated with special truncations of the coefficients of $\mathcal{Z}_{h}$ behaves like a quasipolynomial. And finally it turns out that the evaluation of the quasipolynomial at the origin (this value is called the 'periodic constant $\operatorname{pc}\left(\mathcal{Z}_{h}\right)$ of $\mathcal{Z}_{h}$ ') provides the Seiberg-Witten invariant associated with the corresponding $\sin ^{c}$-structure indexed by $h$. (For details see Sect. 2.)

This is a rather complex regularization procedure. Usually it is hard to find the quasipolynomial, one needs to know all the coefficients of $Z_{h}$ and to understand their asymptotic behaviour.

In fact, there exists an expected reformulation/generalization as follows. Particular examples and families suggest that there must exists an object which is even more general and guides the above periodic constant computation as well: one predicts a unique canonical decomposition of $\mathcal{Z}_{h}$ into a sum $\mathcal{Z}_{h}^{\text {neg }}+P_{h}^{+}$such that $\mathcal{Z}_{h}^{\text {neg }}$ is a rational function 'with negative degree' (or, with zero periodic constant) and $P_{h}^{+}$a finite polynomial, such that $P_{h}^{+}(1)=\operatorname{pc}\left(\mathcal{Z}_{h}\right)$. In this way, one finds a multivariable polynomial generalization of the Seiberg-Witten invariants, namely $P_{h}^{+}$. 
The first goal of the present article is to provide from $\mathcal{Z}(\mathbf{t})$ a simple expression for $\operatorname{pc}\left(\mathcal{Z}_{h}\right)$, or for the Seiberg-Witten invariants of the link. This is done using combination of two dualities: one of them is the topological trace of a Gorenstein type duality, the second is the Ehrhart-Macdonald-Stanley duality of Ehrhart theory. It turns out that $\operatorname{pc}\left(\mathcal{Z}_{h}\right)$ is an easy precise sum of coefficients of the 'dual' series $\mathcal{Z}_{\left[Z_{K}\right]-h \text {, where }}$ $\left[Z_{K}\right]-h \in H$ is the Gorenstein type dual of $h$. The second goal is to make the connection with Ehrhart theory deeper: in this way we show that the decomposition $\mathcal{Z}_{h}^{\text {neg }}+P_{h}^{+}$exists indeed, and we determine $P_{h}^{+}$in language of lattice points of some polytopes. In this way we get such an expressions for the Seiberg-Witten invariants as well.

\section{2}

Let us provide a more detailed discussion regarding the connection with lattice polytopes.

The interactions between polyhedral combinatorics and algebraic geometry is a classical and very intensive research theme. The most classical example is the toric geometry which compiles questions of algebraic geometry in terms of combinatorics of convex cones and lattice polytopes. This leads to an infusion of combinatorial methods and formulas into the geometry and topology of complex analytic/algebraic varieties.

In the theory of complex normal surface singularities there are several results which establish the above interaction in the case of hypersurface, or, more generally, of isolated complete intersection singularities, with Newton non-degenerate principal part. For such germs one defines Newton polyhedrons using the nontrivial monomials of the defining equations, from which several invariants are expressed, see e.g. [2,4,6, $18,19,27,29,42,43,46,49]$.

From a purely topological point of view, a formula by [15] provides the Casson invariant of a Brieskorn homology sphere (as the Euler characteristic of instanton Floer homology) in terms of counting lattice points inside a tetrahedron in $\mathbb{R}^{3}$ (see also [38]). In fact, this lattice point counting formula can also be generalized to Seifert homology spheres with at most 4 singular fibers [40]. Note that for rational homology sphere 3-manifolds the Seiberg-Witten invariant is the generalization of the Casson invariant. Furthermore, for links of Newton non-degenerate hypersurface germs the Seiberg-Witten invariant can be equated with the geometric genus of the singularity proven by [46], for which a lattice point counting formula in terms of the Newton polyhedron is given by [27]. However, this family of germs is rather restrictive and does not give (even the idea) how to find topologically the polytopes in general. Thus, the following goal is very natural:

Problem: Find an explicit lattice point counting interpretation of the SeibergWitten invariant using certain 'topological' polytopes associated with the link.

In this direction a new channel has been opened in the work of the first and the last author [20] by developing a connection between the Seiberg-Witten invariant of rational homology sphere links and equivariant multivariable Ehrhart theory of dilated polytopes, introduced in [20, Section 3] by generalizing results of Ehrhart 
[12], McMullen [28] and Beck [3]. It has been shown that equivariant multivariable Ehrhart quasipolynomials (endowed with their Ehrhart-Macdonald-Stanley reciprocity $[13,26,47]$ ) appear naturally in the study of multivariable rational 'zeta functions' using the coefficient function and the periodic constant of their Taylor expansion at the origin. However, in that work the strength of the reciprocity and its geometric reinterpretations were not exploited totally. Though this connection suggested the existence of a 'polynomial-negative degree part' decomposition of multivariable topological Poincaré series, hence a polynomial generalization of the Seiberg-Witten invariant, and it generated an intense activity (see e.g. [20,22,23]), the complete explanation waited till the present manuscript.

\section{3}

The final output of the present paper is a complete answer to the Problem formulated above. The main result transforms the equivariant Ehrhart-Macdonald-Stanley reciprocity to the level of series, giving a duality between two objects: the periodic constant of the Taylor expansion at the origin and a finite sum of coefficients of the Taylor expansion at infinity.

This, applied to the multivariable topological Poincaré series of normal surface singularities, gives rise to an identification of the Seiberg-Witten invariants indexed by $h$ with a finite sum of coefficients of the 'dual' series indexed by $\left(\left[Z_{K}\right]-h\right)$. (Here $-Z_{K}$ is the cycle of the dualizing sheaf.) This can be regarded as an extension of the $Z_{K}$-symmetry given by the Riemann-Roch formula for the Euler characteristic of line bundles on the resolution. One can also be viewed as a combinatorial analog of the Laufer's duality for equivariant geometric genera of the singularity.

Moreover, it turns out that this duality at the level of series provides the wished multivariable 'polynomial-negative degree part' decomposition of the Poincaré series as well. It also motivates the definition of the topological polytopes associated with a plumbing graph of any rational homology sphere link, which provides an inclusionexclusion lattice point counting formula for the Seiberg-Witten invariants, similar to the one of Khovanskii [18] and Morales [29] for the geometric genus of Newton non-degenerate isolated complete intersection singularities.

\section{4}

Technically, some of the main results can be formulated as follows. Let $\mathcal{V}, \mathcal{N}$ and $\mathcal{E}$ denote the set of vertices, nodes and end-vertices of the graph $\Gamma$. Let $L$ be the lattice associated with $\Gamma$ with the natural fixed basis $\left\{E_{v}\right\}_{v \in \mathcal{V}}$, and let $L^{\prime}$ be the dual lattice. Write $H:=L^{\prime} / L \simeq H_{1}(M, \mathbb{Z})$, where $M$ is the plumbed 3-manifold associated with $\Gamma$. Denote $r_{h}=\sum_{v} l_{v}^{\prime} E_{v} \in L^{\prime}$ the unique representative of $h$ (that is, $h=\left[r_{h}\right] \in L^{\prime} / L$ ) with all $l_{v}^{\prime} \in \mathbb{Q} \cap[0,1)$. For $l^{\prime}=\sum_{v} l_{v}^{\prime} E_{v}$ and $l^{\prime \prime}=\sum_{v} l_{v}^{\prime \prime} E_{v}$ we write $l^{\prime} \geq l^{\prime \prime}$ (resp. $l^{\prime} \prec l^{\prime \prime}$ ) if $l_{v}^{\prime} \geq l_{v}^{\prime \prime}$ (resp. $l_{v}^{\prime}<l_{v}^{\prime \prime}$ ) for all $v \in \mathcal{V}$. (Note that $¥$ differs from $\prec$.)

We set the antidual basis $\left\{E_{v}^{*}\right\}_{v \in \mathcal{V}}$ and the rational function $f(\mathbf{t}):=\prod_{v \in \mathcal{V}}(1-$ $\left.\mathbf{t}^{E_{v}^{*}}\right)^{\delta_{v}-2}$, where $\delta_{v}$ is the valency of the vertex $v$ in $\Gamma$, and $\mathbf{t}^{l^{\prime}}:=\prod_{v} t_{v}^{l_{v}^{\prime}}$ where 
$l^{\prime}=\sum_{v} l_{v}^{\prime} E_{v}$. Let $\mathcal{Z}(\mathbf{t})=\sum_{l^{\prime} \in L^{\prime}} z\left(l^{\prime}\right) \mathbf{t}^{l^{\prime}}$ be its Taylor expansion at the origin. $\mathcal{Z}(\mathbf{t})$ decomposes as $\sum_{h \in H} \mathcal{Z}_{h}(\mathbf{t})$, where $\mathcal{Z}_{h}(\mathbf{t}):=\sum_{\left[l^{\prime}\right]=h} z\left(l^{\prime}\right) \mathbf{t}^{l^{\prime}}$. Furthermore, for any

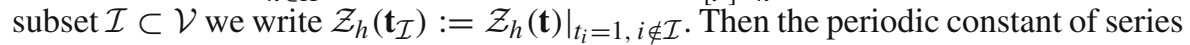
$\mathcal{Z}_{h}(\mathbf{t})$ and $\mathcal{Z}_{h}\left(\mathbf{t}_{\mathcal{N}}\right)$ (defined via the regularization procedure) satisfy:

Theorem A $[20,34] \operatorname{pc}\left(\mathcal{Z}_{h}(\mathbf{t})\right)=\operatorname{pc}\left(\mathcal{Z}_{h}\left(\mathbf{t}_{\mathcal{N}}\right)\right)=-\mathfrak{s w}_{h}^{\text {norm }}(M)$, where $\mathfrak{s w}_{h}^{\text {norm }}(M)$ is the normalized Seiberg-Witten invariant of $M$ associated with $h \in H$ (via the $H$ torsor action of $H$ on $\operatorname{Spin}^{c}(M)$ ). (For details see Theorems 2.4.2 and 4.1.1 below.) $\mathcal{Z}\left(\mathbf{t}_{\mathcal{I}}\right)$

We fix $h \in H$ and $\mathcal{I} \subset \mathcal{V}$ and define the counting function of the coefficients of

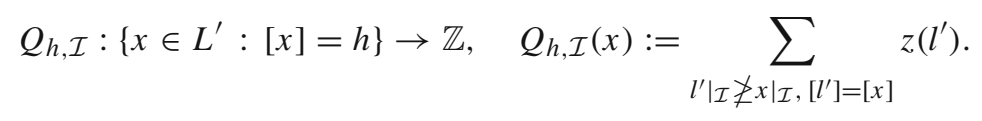

Above the sum is finite since $\mathcal{Z}$ is supported in the (positive) Lipman cone $\mathcal{S}^{\prime}:=$ $\mathbb{Z}_{\geq 0}\left\langle E_{v}^{*}\right\rangle_{v}$

Theorem B (Theorem 4.4.1 in the text) For any $\mathcal{I} \subset \mathcal{V}, \mathcal{I} \neq \emptyset$ and $h \in H$ one has $\operatorname{pc}\left(\mathcal{Z}_{h}\left(\mathbf{t}_{\mathcal{I}}\right)\right)=Q_{\left[Z_{K}\right]-h, \mathcal{I}}\left(Z_{K}-r_{h}\right)$. In other words, the periodic constant of $\mathcal{Z}_{h}\left(\mathbf{t}_{\mathcal{I}}\right)$ can be determined as a finite sum of coefficients of the dual series $\mathcal{Z}_{\left[Z_{K}\right]-h}\left(\mathbf{t}_{\mathcal{I}}\right)$.

For any $\mathcal{I}$ and $h$ as above we write $\mathbf{t}_{\mathcal{I}}^{Z_{K}-E} \mathcal{Z}_{\left[Z_{K}\right]-h}\left(\mathbf{t}_{\mathcal{I}}^{-1}\right)$ as $\sum_{l^{\prime} \in Z_{K}-E-\mathcal{S}^{\prime}} w\left(l^{\prime}\right) \mathbf{t}_{\mathcal{I}}^{l^{\prime}}$ and define

$$
P_{h, \mathcal{I}}^{+}\left(\mathbf{t}_{\mathcal{I}}\right):=\sum_{l^{\prime}|\mathcal{I} \nprec 0|_{\mathcal{I}}} w\left(l^{\prime}\right) \mathbf{t}_{\mathcal{I}}^{l^{\prime}}, \quad f_{h, \mathcal{I}}^{n e g}\left(\mathbf{t}_{\mathcal{I}}\right):=\sum_{\left.\left.l^{\prime}\right|_{\mathcal{I}} \prec 0\right|_{\mathcal{I}}} w\left(l^{\prime}\right) \mathbf{t}_{\mathcal{I}}^{l^{\prime}} .
$$

Set also $P_{h, \mathcal{I}}^{+}(\mathbf{1}):=\left.P_{h, \mathcal{I}}^{+}\left(\mathbf{t}_{\mathcal{I}}\right)\right|_{t_{i}=1, \forall i}$.

Theorem $\mathrm{C}$ (Theorem 5.3.2 in the text) The decomposition $f_{h}\left(\mathbf{t}_{\mathcal{I}}\right)=P_{h, \mathcal{I}}^{+}\left(\mathbf{t}_{\mathcal{I}}\right)+$ $f_{h, \mathcal{I}}^{n e g}\left(\mathbf{t}_{\mathcal{I}}\right)$ is the 'polynomial-negative degree part' decomposition of $f_{h}\left(\mathbf{t}_{\mathcal{I}}\right)$. In particular, $P_{h, \mathcal{I}}^{+}(\mathbf{1})=\operatorname{pc}\left(\mathcal{Z}_{h}\left(\mathbf{t}_{\mathcal{I}}\right)\right)$.

For any $l^{\prime} \in L^{\prime}$ and $v \in \mathcal{V}$ define the polytope $\mathcal{P}_{v}^{\left(l^{\prime}\right)}=\left\{\mathbf{y} \in \mathbb{R}_{\geq 0}^{|\mathcal{E}|}:\left.\sum_{e \in \mathcal{E}} y_{e} E_{e}^{*}\right|_{v} \leq\right.$ $\left.l_{v}^{\prime}\right\}$. Similarly, for any $\mathcal{I} \subset \mathcal{V}$ define also the 'concave polytope'

$$
\widetilde{\mathcal{P}}_{\mathcal{I}}^{\left(l^{\prime}\right)}=\bigcup_{v \in \mathcal{I}} \mathcal{P}_{v}^{\left(l^{\prime}\right)} \subset \mathbb{R}_{\geq 0}^{|\mathcal{E}|}
$$

Using this we define a series of polytopes as follows.

Let us write the set of nodes as $\mathcal{N}=\left\{v_{1}, \ldots, v_{s}\right\}$. Then consider the multiset $\mathcal{N}^{m}:=\left\{v_{1}^{\delta_{v_{1}}-2}, \ldots, v_{s}^{\delta_{v_{s}}-2}\right\}$, where in $\mathcal{N}^{m}$ each node $v$ has multiplicity $\delta_{v}-2$. Let $\mathcal{I}^{m} \subset \mathcal{N}^{m}$ be a non-trivial sub-multiset, $\mathcal{I}^{m}=\left\{v_{i_{1}}^{k_{i_{1}}}, \ldots, v_{i_{r}}^{k_{i_{r}}}\right\}$, where $\left\{v_{i_{1}}, \ldots, v_{i_{r}}\right\}=$ $\mathcal{I} \subset \mathcal{N}$ and $0<k_{i_{j}} \leq \delta_{v_{i}}-2$ for all $j$, and $0<r \leq s$. To such $\mathcal{I}^{m}$ we define 
$\left|\mathcal{I}^{m}\right|:=\sum_{j} k_{i_{j}}$, the cycle $l^{\prime}\left(\mathcal{I}^{m}\right)=\sum_{j} k_{i_{j}} E_{v_{i_{j}}}^{*},\left(\begin{array}{c}\delta-2 \\ k_{\mathcal{I}^{m}}\end{array}\right):=\prod_{j}\left(\begin{array}{c}\delta_{v_{j}}-2 \\ k_{i_{j}}\end{array}\right)$, and the polytope $\widetilde{\mathcal{P}}_{\mathcal{I}^{m}}:=\widetilde{\mathcal{P}}_{\mathcal{I}}^{\left(l^{\prime}\left(\mathcal{I}^{m}\right)\right)}$.

Theorem D (Theorem 6.2.5 in the text) For polytope of type $\widetilde{\mathcal{P}}_{\mathcal{I}}^{\left(l^{\prime}\right)}$ let $R_{h}\left(\widetilde{\mathcal{P}}_{\mathcal{I}}^{\left(l^{\prime}\right)}\right)$ be the number of integral points in $\widetilde{\mathcal{P}}_{\mathcal{I}}^{\left(l^{\prime}\right)}$ with class $\left[\sum_{e} y_{e} E_{e}^{*}\right]=\left[l^{\prime}\right]-h$ and all coordinates strictly positive. Then

$$
-\mathfrak{s w}_{h}^{\text {norm }}(M)=\sum_{\emptyset \neq \mathcal{I}^{m} \subset \mathcal{N}^{m}}(-1)^{|\mathcal{E}|-\left|\mathcal{I}^{m}\right|} \cdot\left(\begin{array}{l}
\delta-2 \\
k_{\mathcal{I}^{m}}
\end{array}\right) \cdot R_{h}\left(\widetilde{\mathcal{P}}_{\mathcal{I}^{m}}\right)
$$

\section{5}

The organization of the paper is the following. Section 2 contains preliminaries about plumbing graphs, manifolds, their Seiberg-Witten invariants, and also Poincaré series and their counting functions and periodic constants. In Sect. 3 we shortly review the equivariant multivariable Ehrhart theory from [20,22], then we formulate the EhrhartMacdonald-Stanley duality for rational functions. Section 4 contains the study of the duality for multivariable topological Poincaré series and the identification of the Seiberg-Witten invariants with finite sum of certain coefficients of the dual series. Section 5 describes the 'polynomial-negative degree part' decomposition. It contains a brief motivation and history of the problem, and some examples as well. Lastly, in Sect. 6 we define the topological polytopes and we prove the lattice point counting formula for the polynomial part of the Poincaré series, in particular, for the SeibergWitten invariants. The results of this section are illustrated on a concrete example in Example 6.2.15. We also discuss the similarities with the Khovanskii-Morales formulas using Minkowski sums of associated polyhedrons.

\section{Preliminaries}

For more details regarding plumbing graphs, plumbed manifolds and their relations with normal surface singularities see [5,14,30-34,36,39]; for Poincaré series see also $[8,9]$.

\subsection{Plumbing graphs: plumbed 3-manifolds}

We fix a connected plumbing graph $\Gamma$ whose associated intersection matrix is negative definite. We denote the corresponding oriented plumbed 3-manifold by $M=M(\Gamma)$. In this article we always assume that $M$ is a rational homology sphere, equivalently, $\Gamma$ is a tree with all genus decorations zero.

We use the notation $\mathcal{V}$ for the set of vertices, $\delta_{v}$ for the valency of a vertex $v, \mathcal{N}$ for the set of nodes (vertices with $\delta_{v} \geq 3$ ), and $\mathcal{E}$ for the set of end-vertices (vertices with $\left.\delta_{v}=1\right)$. 
Let $\widetilde{X}$ be the plumbed 4-manifold with boundary associated with $\Gamma$, hence $\partial \widetilde{X}=M$. Its second homology $L:=H_{2}(\tilde{X}, \mathbb{Z})$ is a lattice, freely generated by the classes of 2-spheres $\left\{E_{v}\right\}_{v \in \mathcal{V}}$, with a negative definite intersection form (, ). Furthermore, $H^{2}(\widetilde{X}, \mathbb{Z})$ can be identified with the dual lattice $L^{\prime}:=\operatorname{Hom}_{\mathbb{Z}}(L, \mathbb{Z})=\left\{l^{\prime} \in L \otimes \mathbb{Q}\right.$ : $\left.\left(l^{\prime}, L\right) \in \mathbb{Z}\right\} . L^{\prime}$ is generated by the (anti)dual classes $\left\{E_{v}^{*}\right\}_{v \in \mathcal{V}}$ defined by $\left(E_{v}^{*}, E_{w}\right)=$ $-\delta_{v w}$, the opposite of the Kronecker symbol. One has the inclusions $L \subset L^{\prime} \subset L \otimes \mathbb{Q}$, and $H_{1}(M, \mathbb{Z}) \simeq L^{\prime} / L$, denoted by $H$. We write $[x]$ for the class of $x \in L^{\prime}$ in $H$.

For any $h \in H$ let $r_{h}=\sum_{v} l_{v}^{\prime} E_{v} \in L^{\prime}$ be its unique representative with all $l_{v}^{\prime} \in[0,1)$.

We say that $l^{\prime}=\sum_{v} l_{v}^{\prime} E_{v} \geq 0$ if and only if each $l_{v}^{\prime} \geq 0$. This induces a partial ordering on $L^{\prime}: l_{1}^{\prime} \geq \underline{\sim}_{2}^{\prime}$ if and only if $l_{1}^{\prime}-l_{2}^{\prime} \geq 0$.

The 4-manifold $\widetilde{X}$ has a complex structure. In fact, any such $M(\Gamma)$ is the link of a complex normal surface singularity $(X, o)$, which has a resolution $\widetilde{X} \rightarrow X$ with resolution graph $\Gamma$ (see e.g. [30]), and the complex analytic smooth surface $\widetilde{X}$ as a $C^{\infty}$ manifold is the plumbed 4-manifold associated with $\Gamma$. Let $K \in L^{\prime}$ be its canonical cycle. Though the complex structure is not unique, $K$ is determined topologically by $L$ via the adjunction formulae $\left(K+E_{v}, E_{v}\right)+2=0$ for all $v$. In some cases, it is more convenient to use $Z_{K}:=-K \in L^{\prime}$, hence, by adjunction formulae,

$$
Z_{K}-E=\sum_{v \in \mathcal{V}}\left(\delta_{v}-2\right) E_{v}^{*}
$$

where we denote $E:=\sum_{v \in \mathcal{V}} E_{v}$. In the sequel $|A|$ denotes the cardinality of the set $A$.

\subsection{The series $\mathcal{Z}(\mathbf{t})$}

The multivariable topological Poincaré series is the Taylor expansion $\mathcal{Z}(\mathbf{t})=$ $\sum_{l^{\prime}} z\left(l^{\prime}\right) \mathbf{t}^{l^{\prime}} \in \mathbb{Z}\left[\left[L^{\prime}\right]\right]$ at the origin of the rational 'zeta-function'

$$
f(\mathbf{t})=\prod_{v \in \mathcal{V}}\left(1-\mathbf{t}^{E_{v}^{*}}\right)^{\delta_{v}-2},
$$

where $\mathbf{t}^{l^{\prime}}:=\prod_{v \in \mathcal{V}} t_{v}^{l_{v}^{\prime}}$ for any $l^{\prime}=\sum_{v \in \mathcal{V}} l_{v}^{\prime} E_{v} \in L^{\prime}\left(l_{v}^{\prime} \in \mathbb{Q}\right)$, cf. [8,9,33]. Since in the duality discussions (Sect. 3) we will need Taylor expansions of $f$ both at 0 and $\infty$, we prefer to make distinction between $f$ and $\mathcal{Z}$. It decomposes as $\mathcal{Z}(\mathbf{t})=\sum_{h \in H} \mathcal{Z}_{h}(\mathbf{t})$, where $\mathcal{Z}_{h}(\mathbf{t})=\sum_{\left[l^{\prime}\right]=h} z\left(l^{\prime}\right) \mathbf{t}^{l^{\prime}}$. The expression (2.2.1) shows that $\mathcal{Z}(\mathbf{t})$ is supported in the Lipman cone $\mathcal{S}^{\prime}:=\mathbb{Z}_{\geq 0}\left\langle E_{v}^{*}\right\rangle_{v \in \mathcal{V}}$. Since (, ) is negative definite, all the entries of $E_{v}^{*}$ are strict positive, hence $\mathcal{S}^{\prime} \subset\left\{\sum_{v} l_{v}^{\prime} E_{v}: l_{v}^{\prime}>0\right\} \cup\{0\}$. Thus (cf. [34, (2.1.2)] and Fig. 1) for any $x \in L^{\prime}$,

$$
\left\{l^{\prime} \in \mathcal{S}^{\prime}: l^{\prime} \nsupseteq x\right\} \text { is finite. }
$$

Fix $h \in H$. We define a 'counting function' of the coefficients of $\mathcal{Z}_{h}$ by

$$
Q_{h}: L_{h}^{\prime}:=\left\{x \in L^{\prime}:[x]=h\right\} \rightarrow \mathbb{Z}, \quad Q_{h}(x)=\sum_{l^{\prime} \nsupseteq x,\left[l^{\prime}\right]=h} z\left(l^{\prime}\right) .
$$


Fig. 1 The truncation $l^{\prime} \nsupseteq x$

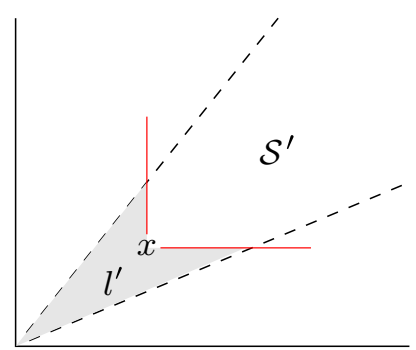

The appearance of this type of truncation $\left\{l^{\prime} \nsucceq x,\left[l^{\prime}\right]=h\right\}$ for the counting function in the above sum is motivated by the results below, see e.g. (2.3.2) (or [35]).

\subsection{Seiberg-Witten invariants of $M$}

We denote by $\operatorname{Spin}^{c}(\widetilde{\widetilde{X}})$ the set of $\operatorname{spin}^{c}$-structures on $\widetilde{X}$ and let $\widetilde{\sigma}_{\text {can }}$ be the canonical spin ${ }^{c}$-structure on $\widetilde{X}$ identified by $c_{1}\left(\widetilde{\sigma}_{c a n}\right)=Z_{K} . \operatorname{Spin}^{c}(\widetilde{X})$ is an $L^{\prime}$-torsor and if we denote the action by $*$, then $c_{1}\left(l^{\prime} * \widetilde{\sigma}\right)=c_{1}(\widetilde{\sigma})+2 l^{\prime}$. All the $\operatorname{spin}^{c}$-structures on $M$ are obtained by restrictions from $\tilde{X}$. $\operatorname{Spin}^{c}(M)$ is an $H$-torsor, compatible with the projection $L^{\prime} \rightarrow H$. The canonical spin ${ }^{c}$-structure on $M$ is the restriction of $\widetilde{\sigma}_{c a n}$.

Further details regarding $\operatorname{spin}^{c}$-structures can be found eg. in [16, page 415].

We denote by $\mathfrak{s w}_{\sigma}(M) \in \mathbb{Q}$ the Seiberg-Witten invariant of $M$ indexed by the $\operatorname{spin}^{c}$-structures $\sigma \in \operatorname{Spin}^{c}(M)$ (cf. [24,41]). (We will use the sign convention of $[5,34]$.)

In the last years, several combinatorial expressions were established for the SeibergWitten invariants. For rational homology spheres, Nicolaescu [41] showed that $\mathfrak{s w}(M)$ is equal to the Reidemeister-Turaev torsion normalized by the Casson-Walker invariant. In the case when $M$ is a negative definite plumbed rational homology sphere, a combinatorial formula for Casson-Walker invariant in terms of the plumbing graph can be found in Lescop [25], and the Reidemeister-Turaev torsion is determined by Némethi and Nicolaescu [36] using Dedekind-Fourier sums.

A different combinatorial formula of $\left\{\mathfrak{s} \mathfrak{w}_{\sigma}(M)\right\}_{\sigma}$ was proved in [34] using qualitative properties of the coefficients of the series $\mathcal{Z}(\mathbf{t})$.

Theorem 2.3.1 [34] For any $l^{\prime} \in-K+\operatorname{int}\left(\mathcal{S}^{\prime}\right)\left(\right.$ where $\left.\operatorname{int}\left(\mathcal{S}^{\prime}\right)=\mathbb{Z}_{>0}\left\langle E_{v}^{*}\right\rangle_{v \in \mathcal{V}}\right)$

$$
-Q_{\left[l^{\prime}\right]}\left(l^{\prime}\right)=\frac{\left(K+2 l^{\prime}\right)^{2}+|\mathcal{V}|}{8}+\mathfrak{s w}_{\left[-l^{\prime}\right] * \sigma_{c a n}}(M) .
$$

If we fix $h \in H$ and we write $l^{\prime}=l+r_{h}$ with $l \in L$, then the right-hand side of (2.3.2) is a multivariable quadratic polynomial in variable $l \in L$, a fact which will be exploited conceptually next.

\subsection{Periodic constants}

One of the key tools of the present article is an invariant associated with series motivated by properties of Hilbert-Samuel functions used in algebraic geometry and singularity 
theory. It creates a bridge with Ehrhart theory and the properties of its quasipolynomials. It is called the periodic constant of the series. For one-variable series they were introduced in [37,44], see also [5], the multivariable generalization is treated in [20].

Let $S(t)=\sum_{l \geq 0} c_{l} t^{l} \in \mathbb{Z}[[t]]$ be a formal power series with one variable. Assume that for some $p \in \mathbb{Z}_{>0}$ the counting function $Q^{(p)}(n):=\sum_{l=0}^{p n-1} c_{l}$ is a polynomial $\mathfrak{Q}^{(p)}$ in $n$. Then the constant term $\mathfrak{Q}^{(p)}(0)$ is independent of $p$ and it is called the periodic constant $\operatorname{pc}(S)$ of the series $S$. E.g., if $S(t)$ is a finite polynomial, then $\operatorname{pc}(S)$ exists and it equals $S(1)$. If the coefficients of $S(t)$ are given by a Hilbert function $l \mapsto c(l)$, which admits a Hilbert polynomial $H(l)$ with $c(l)=H(l)$ for $l \gg 0$, then one shows that $S^{r e g}(t)=\sum_{l \geq 0} H(l) t^{l}$ has zero periodic constant and $\operatorname{pc}(S)=$ $\operatorname{pc}\left(S-S^{r e g}\right)+\operatorname{pc}\left(S^{r e g}\right)=\left(S-S^{r e g}\right)(1)$, measuring the difference between the Hilbert function and Hilbert polynomial.

For the multivariable case we consider a (negative) definite lattice $L=\mathbb{Z}\left\langle E_{v}\right\rangle_{v}$, its dual lattice $L^{\prime}$, a series $S(\mathbf{t}) \in \mathbb{Z}\left[\left[L^{\prime}\right]\right]$ (e.g. $\mathcal{Z}(\mathbf{t})$ ), and its well-defined counting function $Q_{h}=Q_{h}(S(\mathbf{t}))$ as in (2.2.3) for fixed $h \in L^{\prime} / L$. (In fact, the definition extends to the case of any two free $\mathbb{Z}$-modules $L \subset L^{\prime}$ of the same rank with quotient group denoted by $H:=L^{\prime} / L$, the setup of Sect. 3.) Assume that there exist a real cone $\mathcal{K} \subset L^{\prime} \otimes \mathbb{R}$ whose affine closure is top-dimensional, $l_{*}^{\prime} \in \mathcal{K}$, a sublattice $\widetilde{L} \subset L$ of finite index, and a quasipolynomial (ie. a polynomial whose coefficients are periodic functions) $\mathfrak{Q}_{h}(l)(l \in \widetilde{L})$ (written also as $\left.\mathfrak{Q}_{h}^{\mathcal{K}}(l)\right)$ such that $Q_{h}\left(l+r_{h}\right)=\mathfrak{Q}_{h}(l)$ for any $l+r_{h} \in\left(l_{*}^{\prime}+\mathcal{K}\right) \cap\left(\widetilde{L}+r_{h}\right)$. Then we say that the counting function $Q_{h}$ (or just $S_{h}(\mathbf{t})$ ) admits a quasipolynomial in $\mathcal{K}$, namely $\mathfrak{Q}_{h}(l)$, and also an (equivariant, multivariable) periodic constant associated with $\mathcal{K}$, which is defined by

$$
\operatorname{pc}^{\mathcal{K}}\left(S_{h}(\mathbf{t})\right):=\mathfrak{Q}_{h}(0)
$$

The definition does not depend on the choice of the sublattice $\widetilde{L}$, which corresponds to the choice of $p$ in the one-variable case. This is responsible for the name 'periodic' in the definition. The definition is independent of the choice of $l_{*}^{\prime}$ as well.

By the combinatorial stability results, cf. [20, Section 4.3.7] or [22,48], of multivariable Ehrhart-type quasipolynomials (counting lattice points with special coefficients in polytopes attached to $\mathcal{Z}(\mathbf{t})$ ), one can construct a conical chamber decomposition of the space $L^{\prime} \otimes \mathbb{R}$, such that each cone satisfies the above definition (hence provides a periodic constant). This decomposition, in principle, divides $\mathcal{S}_{\mathbb{R}}^{\prime}:=\mathbb{R}_{\geq 0}\left\langle E_{v}^{*}\right\rangle_{v \in \mathcal{V}}$ into several sub-cones (hence, providing different quasipolynomials and periodic constants associated with these sub-cones of $\mathcal{S}_{\mathbb{R}}^{\prime}$ ). However, Theorem 2.3.1 guarantees that this is not the case, the whole $\mathcal{S}_{\mathbb{R}}^{\prime}$ admits a unique quasipolynomial, in particular, a periodic constant (cf. also with [22]). Hence, Theorem 2.3.1 reads as follows.

Theorem 2.4.2 [34] The counting function of $\mathcal{Z}_{h}(\mathbf{t})$ in the cone $S_{\mathbb{R}}^{\prime}$ admits the (quasi)polynomial

$$
\mathfrak{Q}_{h}(l)=-\frac{\left(K+2 r_{h}+2 l\right)^{2}+|\mathcal{V}|}{8}-\mathfrak{s w}_{-h * \sigma_{c a n}}(M),
$$




$$
\mathrm{pc}^{S_{\mathbb{R}}^{\prime}}\left(\mathcal{Z}_{h}(\mathbf{t})\right)=\mathfrak{Q}_{h}(0)=-\mathfrak{s w}_{-h * \sigma_{c a n}}(M)-\frac{\left(K+2 r_{h}\right)^{2}+|\mathcal{V}|}{8} .
$$

The right-hand side of (2.4.4) with opposite sign is called the $r_{h}$-normalized Seiberg-Witten invariant of $M$, and it is denoted by $\mathfrak{s w}_{h}^{\text {norm }}(M)$.

\section{Ehrhart-Macdonald-Stanley duality for rational functions}

\section{1}

We wish to apply equivariant Ehrhart theory for the computation of the periodic constant of the topological Poincaré series. For this we review some results from the Ehrhart theory. We will use a similar setup as in Sect. 2 to make the comparison easier: we will fix a free $\mathbb{Z}$-module $L$, another one $L^{\prime} \supset L$ of the same rank (but in this Ehrhart context $L^{\prime}$ is not necessarily the dual of $L$, in fact $L$ carries no intersection form at all). We write $H$ for $L^{\prime} / L, d$ for the order of $H$, and we fix a basis $\left\{E_{v}\right\}_{v \in \mathcal{V}}$ in $L$.

We consider multivariable rational functions (in variables $\mathbf{t}^{L^{\prime}}$ ) of type

$$
z(\mathbf{t})=\frac{\sum_{k=1}^{r} \iota_{k} \mathbf{t}^{b_{k}}}{\prod_{i=1}^{n}\left(1-\mathbf{t}^{a_{i}}\right)}
$$

where $\left\{\iota_{k}\right\}_{k=1}^{r} \in \mathbb{Z},\left\{b_{k}\right\}_{k=1}^{r},\left\{a_{i}\right\}_{i=1}^{n} \in L^{\prime}$ and for any $l^{\prime}=\sum_{v} l_{v}^{\prime} E_{v} \in L^{\prime}$ we write $\mathbf{t}^{l^{\prime}}=t_{1}^{l_{1}^{\prime}} \ldots t_{s}^{l_{s}^{\prime}}$. (Though $l_{v}^{\prime} \in \mathbb{Q}$ we still call the elements of $\mathbb{Z}\left[L^{\prime}\right]$ 'polynomials', and fractions of 'polynomials' 'rational functions'.) We also assume that all the coordinates $a_{i, v}$ of $a_{i}$ are strict positive.

We consider the Taylor expansion $T z(\mathbf{t})$ of $z(\mathbf{t})$ at the origin

$$
T z(\mathbf{t})=\sum_{l^{\prime}} z\left(l^{\prime}\right) \mathbf{t}^{l^{\prime}} \in \mathbb{Z}\left[\left[\mathbf{t}^{1 / d}\right]\right]\left[\mathbf{t}^{-1 / d}\right]:=\mathbb{Z}\left[\left[t_{1}^{1 / d}, \ldots, t_{s}^{1 / d}\right]\right]\left[t_{1}^{-1 / d}, \ldots, t_{s}^{-1 / d}\right],
$$

and also define the Taylor expansion of $z(\mathbf{t})$ at infinity

$$
T^{\infty} z(\mathbf{t})=\sum_{\tilde{l}} r(\tilde{l}) \mathbf{t}^{\tilde{l}} \in \mathbb{Z}\left[\left[\mathbf{t}^{-1 / d}\right]\right]\left[\mathbf{t}^{1 / d}\right] .
$$

$T^{\infty} z$ is obtained by the substitution $\mathbf{s}=1 / \mathbf{t}$ into the Taylor expansion at $\mathbf{s}=0$ of the function $z(1 / \mathbf{s})$. E.g., if $z(t)=t^{2} /(1-t)$, then $T^{\infty} z(t)=-t\left(1+t^{-1}+t^{-2}+\cdots\right)$. Note that $\sum_{\tilde{l} \geq 0} r(\tilde{l})$ is a finite sum.

\section{2}

The function $z$ has equivariant decomposition $\sum_{h} z_{h}(h \in H)$ with respect to $H=$ $L^{\prime} / L$, where $z_{h}(\mathbf{t})$ is rational of form $\sum_{b^{\prime} \in h+L} \iota_{b^{\prime}} \mathbf{t}^{b^{\prime}} / \prod_{i=1}^{n}\left(1-\mathbf{t}^{|H| a_{i}}\right)\left(\iota_{b^{\prime}} \neq 0\right.$ for 
finite $\left.b^{\prime}\right)$. While the decompositions $\sum_{h} T z_{h}$ and $\sum_{h}\left(T^{\infty} z\right)_{h}$ of the series $T z$ and $T^{\infty} z$ are defined similarly as the decomposition of $\mathcal{Z}$ in 2.1. (Note that the operation $T z \mapsto T^{\infty} z$, defined via the original $z$, preserves all the $h$-components.)

We also might eliminate some of the variables: for any subset $\mathcal{I} \subset \mathcal{V}$ we substitute $t_{i}=1$ in $z(\mathbf{t})$ for all $i \notin \mathcal{I}$; in this way we obtain $z\left(\mathbf{t}_{\mathcal{I}}\right)$. We call this procedure 'reduction'. This procedure at the level of $T z$ is a summation of some of the coefficients. Since the series associated with the denominator of $z$ is supported in the cone $\mathbb{Z}_{\geq 0}\left\langle a_{i}\right\rangle$, the summation is finite.

Note that the $H$-decomposition of the restricted functions are not well-defined. That is, from the restricted function $z\left(\mathbf{t}_{\mathcal{I}}\right):=\left.z(\mathbf{t})\right|_{t_{i}=1, i \notin \mathcal{I}}$ in general one cannot recover anymore the restriction of the $H$-component $z_{h}(\mathbf{t})$. (That is, from the exponent of $\mathbf{t}_{\mathcal{I}}^{l^{\prime}}$ one cannot recover $\left[l^{\prime}\right] \in H$. Here, and in the sequel, for any $l^{\prime} \in L^{\prime}$ we write $\mathbf{t}_{\mathcal{I}}^{l^{\prime}}$ for $\prod_{v \in \mathcal{I}} t_{v}^{l_{v}^{\prime}}$.) Hence, in the presence of decomposition and reduction the only welldefined object is $\left.\left(z_{h}\right)\right|_{t_{i}=1, i \notin \mathcal{I}}$, the reduction of $z_{h}$, which will be denoted by $z_{h}\left(\mathbf{t}_{\mathcal{I}}\right)$. Furthermore, if $\pi_{\mathcal{I}}$ is the natural projection associated with the reduction (elimination of the $\mathcal{V} \backslash \mathcal{I}$-components), then $\pi_{\mathcal{I}}\left(L^{\prime}\right) / \pi_{\mathcal{I}}(L)$ usually is not isomorphic to $H$. Hence, we keep $H$ as an index set and we never consider $\pi_{\mathcal{I}}\left(L^{\prime}\right) / \pi_{\mathcal{I}}(L)$.

\section{3}

We fix $h \in H$ and $\mathcal{I} \subset \mathcal{V}$. We define two functions associated with the coefficients of $T z_{h}\left(\mathbf{t}_{\mathcal{I}}\right)$ : the first is called the counting function (cf. (2.2.3))

$$
Q_{h, \mathcal{I}}: L_{h}^{\prime}:=\left\{x \in L^{\prime}:[x]=h\right\} \rightarrow \mathbb{Z}, \quad Q_{h, \mathcal{I}}(x):=\sum_{\left.l^{\prime}\right|_{\mathcal{I}} \geq\left. x\right|_{\mathcal{I}},\left[l^{\prime}\right]=[x]} z\left(l^{\prime}\right) .
$$

Note that the above sum is finite by strict positivity assumption on the coordinates $a_{i, v}$ of $a_{i}$. The definition selects only the coefficients of $T z_{h}\left(\mathbf{t}_{\mathcal{I}}\right)$, hence if we write $T z_{h}\left(\mathbf{t}_{\mathcal{I}}\right)$ as $\sum_{k^{\prime} \in \pi_{\mathcal{I}} L^{\prime}} z^{(h)}\left(k^{\prime}\right) \mathbf{t}_{\mathcal{I}}^{k^{\prime}}$, then $Q_{h, \mathcal{I}}(x)=\sum_{\left.k^{\prime} \nsupseteq x\right|_{\mathcal{I}}} z^{(h)}\left(k^{\prime}\right)$. It depends only on $\left.x\right|_{\mathcal{I}}$. This truncation and counting function does not (naturally) appear in Ehrhart theory, but this is what is imposed by Theorem 2.3.1.

Our second function is called the modified counting function; it is defined by

$$
q_{h, \mathcal{I}}: L_{h}^{\prime} \rightarrow \mathbb{Z}, \quad q_{h, \mathcal{I}}(x):=\sum_{\left.\left.l^{\prime}\right|_{\mathcal{I}} \prec x\right|_{\mathcal{I}},\left[l^{\prime}\right]=[x]} z\left(l^{\prime}\right),
$$

where for any $a, b \in \mathbb{R}^{|\mathcal{I}|}$ we say that $a \prec b$ if $a_{v}<b_{v}$ for all $v \in \mathcal{I}$. Similarly as above, one also has $q_{h, \mathcal{I}}(x)=\left.\left.\sum k^{\prime}\right|_{\mathcal{I}} \prec x\right|_{\mathcal{I}} z^{(h)}\left(k^{\prime}\right)$. For any $h$ and $\mathcal{I}$ by inclusionexclusion principle

$$
Q_{h, \mathcal{I}}(x)=\sum_{\emptyset \neq \mathcal{J} \subset \mathcal{I}}(-1)^{|\mathcal{J}|+1} q_{h, \mathcal{J}}(x)
$$


This modified counting function will be related to the usual counting functions from the Ehrhart theory. It satisfies several useful properties (e.g. convexity, reciprocity, see below).

\subsection{Ehrhart theory of polytopes associated with the denominator of $z$}

In this subsection we follow the theory developed in [20,22]. Associated with the vectors $\left\{a_{i}\right\}_{i=1}^{n}$ (the exponents in the denominator of $\left.z(\mathbf{t})\right)$ we define two objects. Firstly, let $\mathfrak{l}: \mathbb{R}^{n} \rightarrow L^{\prime} \otimes \mathbb{R}$ be the map given by $\mathfrak{l}(\mathbf{y})=\sum_{i=1}^{n} y_{i} a_{i}$ and consider the representation $\rho: \mathbb{Z}^{n} \rightarrow H$ defined by the composition $\mathbb{Z}^{n} \stackrel{\mathfrak{l}_{\mathbb{Z}^{n}}}{\longrightarrow} L^{\prime} \rightarrow L^{\prime} / L$.

Then, the vectors $\left\{a_{i}\right\}_{i=1}^{n}$ and any $\mathcal{I} \subset \mathcal{V}, \mathcal{I} \neq \emptyset$ (which might vary, cf. (3.3.3)) determine the family of closed dilated convex polytopes with dilatation parameter $l^{\prime}=\sum_{v} l_{v}^{\prime} E_{v} \in L^{\prime}$

$$
\mathcal{P}_{\mathcal{I}}^{\left(l^{\prime}\right)}:=\left\{\mathbf{y} \in\left(\mathbb{R}_{\geq 0}\right)^{n}: \sum_{i} y_{i} a_{i, v} \leq l_{v}^{\prime} \text { for all } v \in \mathcal{I}\right\} .
$$

$\mathcal{P}_{\mathcal{I}}^{\left(l^{\prime}\right)}$ depends only on $l_{\mathcal{I}}^{\prime}:=\left.l^{\prime}\right|_{\mathcal{I}}$. We denote by $\mathcal{F}_{\mathcal{I}}^{\left(l^{\prime}\right)}$ the set of facets of $\mathcal{P}_{\mathcal{I}}^{\left(l^{\prime}\right)}$.

By varying $l_{\mathcal{I}}^{\prime}$ in some region we say that the polytopes $\mathcal{P}_{\mathcal{I}}^{\left(l^{\prime}\right)}$ remain combinatorially stable (or preserve their combinatorial type) if they are equivalent up to homeomorphisms, which preserve the stratification of the faces. By the results of $[22,48]$, the variation of the combinatorial type of $\mathcal{P}_{\mathcal{I}}^{\left(l^{\prime}\right)}$ is determined by the following chamber decomposition of $\pi_{\mathcal{I}}\left(L^{\prime} \otimes \mathbb{R}\right)=\mathbb{R}^{|\mathcal{I}|}$ : let $\mathcal{B}_{\mathcal{I}}$ be the set of all bases $\sigma \subset\left\{\left.a_{i}\right|_{\mathcal{I}},\left.E_{v}\right|_{\mathcal{I}}: i \in\{1, \ldots, n\}, v \in \mathcal{I}\right\}$ of $\mathbb{R}^{|\mathcal{I}|}$. Then a (big, open) chamber $\mathfrak{c}$ is a connected component of $\mathbb{R}^{|\mathcal{I}|} \backslash \cup_{\sigma \in \mathcal{B}_{\mathcal{I}}} \partial \mathbb{R}_{\geq 0} \sigma$, where $\partial \mathbb{R}_{\geq 0} \sigma$ is the boundary of the closed cone $\mathbb{R}_{\geq 0} \sigma$.

Then $\mathcal{P}_{\mathcal{I}}^{\left(l^{\prime}\right)}$ stays combinatorially stable if $l_{\mathcal{I}}^{\prime}$ moves in such a chamber $\mathfrak{c}$. In such a case we can associate to the stable combinatorial type the set (dilated family) of facets $\mathcal{F}_{\mathcal{I}}^{\left(l^{\prime}\right)}$. Moreover, any choice of a subset of facets in a fixed stable topological type provides a 'stable' (dilated) subset of facets $\mathcal{G}_{\mathcal{I}}^{\left(l^{\prime}\right)}$ in each $\mathcal{F}_{\mathcal{I}}^{\left(l^{\prime}\right)}$; we denote this choice by $\mathcal{G}_{\mathcal{I}} \subset \mathcal{F}_{\mathcal{I}}$. Furthermore, for any $h \in H$ and choice $\mathcal{G}_{\mathcal{I}} \subset \mathcal{F}_{\mathcal{I}}$ we consider the counting function of specially chosen lattice points identified by

$$
\mathcal{Q}_{h, \mathcal{G}_{\mathcal{I}}}\left(l^{\prime}\right):=\text { cardinality of }\left(\left(\mathcal{P}_{\mathcal{I}}^{\left(l^{\prime}\right)} \backslash \mathcal{G}_{\mathcal{I}}^{\left(l^{\prime}\right)}\right) \cap \rho^{-1}(h)\right) \text {. }
$$

According to the equivariant Ehrhart theory [20, Theorem 3.1.2(a)], applied to the dilated polytopes $\mathcal{P}_{\mathcal{I}}^{\left(l^{\prime}\right)}$ (parametrized by chamber $\mathfrak{c}$ ), and for any $h \in H$ and $\mathcal{G}_{\mathcal{I}}$, the counting function $\mathcal{Q}_{h, \mathcal{G}_{\mathcal{I}}}\left(l_{\mathcal{I}}^{\prime}\right)$ is a quasipolynomial, denoted by $\mathcal{Q}_{h, \mathcal{G}_{\mathcal{I}}}^{\mathfrak{c}}$.

The above construction shows that the chamber decomposition is independent of the choices of $h \in H$ and $\mathcal{G}_{\mathcal{I}} \subset \mathcal{F}_{\mathcal{I}}$.

One can extend $\mathcal{Q}_{h, \mathcal{G}_{\mathcal{I}}}^{\mathfrak{c}}$ continuously to the closure $\overline{\mathfrak{c}}$ of $\mathfrak{c}$ as a quasipolynomial; all these extensions glue together to a continuous piecewise quasipolynomial of $\mathbb{R}^{|\mathcal{I}|}$. This piecewise quasipolynomial is the expression $\mathcal{Q}_{h, \mathcal{G}_{\mathcal{I}}}$ from (3.4.2). (This means 
that $\mathcal{Q}_{h, \mathcal{G}_{\mathcal{I}}}\left(l_{\mathcal{I}}^{\prime}\right)$ in terms of $\left\{\mathcal{Q}_{h, \mathcal{G}_{\mathcal{I}}}^{\mathfrak{c}}\right\}_{\mathfrak{c}}$ can be redefined as follows: for any $l_{\mathcal{I}}^{\prime} \in \mathbb{R}^{|\mathcal{I}|}$ first find a chamber $\mathfrak{c}$ such that $l_{\mathcal{I}}^{\prime} \in \overline{\mathfrak{c}}$ and then set $\mathcal{Q}_{h, \mathcal{G}_{\mathcal{I}}}\left(l_{\mathcal{I}}^{\prime}\right):=\mathcal{Q}_{h, \mathcal{G}_{\mathcal{I}}}^{\mathfrak{c}}\left(l_{\mathcal{I}}^{\prime}\right)$.)

By the equivariant Ehrhart-Macdonald-Stanley reciprocity law [20, Theorem 3.1.2(b)], for any fixed $h, \mathcal{G}_{\mathcal{I}} \subset \mathcal{F}_{\mathcal{I}}$ and chamber $\mathfrak{c}$ with $l_{\mathcal{I}}^{\prime} \in \mathfrak{c}$ one has

$$
\mathcal{Q}_{h, \mathcal{G}_{\mathcal{I}}}^{\mathfrak{c}}\left(l_{\mathcal{I}}^{\prime}\right)=(-1)^{n} \cdot \mathcal{Q}_{-h, \mathcal{F}_{\mathcal{I}} \backslash \mathcal{G}_{\mathcal{I}}}\left(-l_{\mathcal{I}}^{\prime}\right)
$$

(We warn the reader that usually the parameter $-l_{\mathcal{I}}^{\prime}$ is included in a different chamber than $\mathfrak{c}$, that is $\mathcal{Q}_{-h, \mathcal{F}_{\mathcal{I}} \backslash \mathcal{G}_{\mathcal{I}}}\left(-l_{\mathcal{I}}^{\prime}\right) \neq \mathcal{Q}_{-h, \mathcal{F}_{\mathcal{I}} \backslash \mathcal{G}_{\mathcal{I}}}\left(-l_{\mathcal{I}}^{\prime}\right)$; in (3.4.3) in both sides we use the same chamber $\mathfrak{c}$, and from the pair $l_{\mathcal{I}}^{\prime},-l_{\mathcal{I}}^{\prime}$ only one of them can be in $\overline{\mathfrak{c}}$ provided that $l_{\mathcal{I}}^{\prime} \neq 0$.)

\section{5}

A distinguished subset of facets $\mathcal{G}_{\mathcal{I}} \subset \mathcal{F}_{\mathcal{I}}$ is defined as the coordinate facets $\left\{\mathcal{P}_{\mathcal{I}}^{\left(l^{\prime}\right)} \cap\right.$ $\left.\left\{y_{i}=0\right\}\right\}_{i=1}^{n}$. We denote it by $\mathcal{G}_{\mathcal{I}}^{c o}$.

Then the theory above has the following consequences regarding the modified counting function $q_{h, \mathcal{I}}$ of $T z_{h}\left(\mathbf{t}_{\mathcal{I}}\right)$ defined in (3.3.2):

Corollary 3.5.1 We fix $h$ and $\mathcal{I}$.

(a) $q_{h, \mathcal{I}}$ is a piecewise quasipolynomial, which can be written as

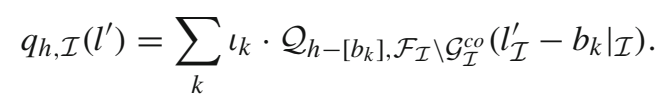

(b) For a fixed chamber $\mathfrak{c}$ of $\mathbb{R}^{|\mathcal{I}|}$ define the quasipolynomial

$$
q_{h, \mathcal{I}}^{\mathfrak{c}}\left(l^{\prime}\right):=\sum_{k} \iota_{k} \cdot \mathcal{Q}_{h-\left[b_{k}\right], \mathcal{F}_{\mathcal{I}} \backslash \mathcal{G}_{\mathcal{I}}^{c o}}^{\mathfrak{c}}\left(l_{\mathcal{I}}^{\prime}-\left.b_{k}\right|_{\mathcal{I}}\right)
$$

Then the restriction of $q_{h, \mathcal{I}}$ to (the closure of $) \cap_{k}\left(\left.b_{k}\right|_{\mathcal{I}}+\mathfrak{c}\right)$ behaves as a quasipolynomial, that is, $q_{h, \mathcal{I}}\left(l^{\prime}\right)=q_{h, \mathcal{I}}^{\mathfrak{c}}\left(l^{\prime}\right)$ for $l_{\mathcal{I}}^{\prime} \in \cap_{k}\left(\left.b_{k}\right|_{\mathcal{I}}+\mathfrak{c}\right)$.

(c) For any fixed chamber $\mathfrak{c}$ the modified counting function $q_{h, \mathcal{I}}$ admits a quasipolynomial in the sense of Sect. 2.4, namely $\mathfrak{q}_{h, \mathcal{I}}^{\mathfrak{c}}$, which satisfies for $l^{\prime}=l+r_{h}$ the identity $\mathfrak{q}_{h, \mathcal{I}}^{\mathfrak{c}}(l)=q_{h, \mathcal{I}}^{\mathfrak{c}}\left(l^{\prime}\right)$, and admits also periodic constant $\mathrm{pc}^{\mathfrak{c}}\left(q_{h, \mathcal{I}}\right)=$ $\mathfrak{q}_{h, \mathcal{I}}^{\mathfrak{c}}(0)=q_{h, \mathcal{I}}^{\mathfrak{c}}\left(r_{h}\right)$ associated with the chamber $\mathfrak{c}$.

This $\operatorname{pc}^{\mathfrak{c}}\left(q_{h, \mathcal{I}}\right)$ will be denoted by $\operatorname{mpc}^{\mathfrak{c}}\left(T z_{h}\left(\mathbf{t}_{\mathcal{I}}\right)\right)$. We call it the modified periodic constant of $T z_{h}\left(\mathbf{t}_{\mathcal{I}}\right)$ associated with $h, \mathcal{I}$ and the chamber $\mathfrak{c}$. (The terminology and the notation emphasize the presence of different cuts in the counting functions.)

\section{6}

In general, the computation of quasipolynomials and their periodic constants (either modified or not) is hard: it measures the asymptotic behaviour of the coefficients in 
a certain cones. The next result based on the equivariant Ehrhart-Macdonald-Stanley reciprocity (3.4.3) shows that (under some conditions) the modified periodic constant associated with a chamber $\mathfrak{c}$ equals a finite sum of certain coefficients of the Taylor expansion at infinity.

Theorem 3.6.1 Fix $h$ and $\mathcal{I}$ as above. Write the $h$-component of the Taylor expansion at infinity as $\left(T^{\infty}\right)_{h}\left(\mathbf{t}_{\mathcal{I}}\right)=\sum_{\tilde{l}} r_{\mathcal{I}}^{(h)}(\tilde{l}) \tilde{t}_{\mathcal{I}}^{\tilde{l}}$. Assume that the chamber $\mathrm{c}$ has the property that $\left.b_{k}\right|_{\mathcal{I}} \in \mathfrak{c}$ for all $k$. Then

$$
\operatorname{mpc}^{\mathfrak{c}}\left(T z_{h}\left(\mathbf{t}_{\mathcal{I}}\right)\right)=\sum_{\tilde{l} \geq 0} r_{\mathcal{I}}^{(h)}(\tilde{l})
$$

Proof By Corollary 3.5.1 the function $q_{h, \mathcal{I}}$ on the set $\cap_{k}\left(\left.b_{k}\right|_{\mathcal{I}}+\mathfrak{c}\right)$ is $\sum_{k} \iota_{k}$. $\mathcal{Q}_{h-\left[b_{k}\right], \mathcal{F}_{\mathcal{I}} \backslash \mathcal{G}_{\mathcal{I}}^{c o}}\left(l_{\mathcal{I}}^{\prime}-b_{k} \mid \mathcal{I}\right)$. Hence, by definitions, $\operatorname{mpc}^{\mathfrak{c}}\left(T z_{h}\left(\mathbf{t}_{\mathcal{I}}\right)\right)=\operatorname{pc}^{\mathfrak{c}}\left(q_{h, \mathcal{I}}\right)$ exists and equals

$$
\sum_{k} \iota_{k} \cdot \mathcal{Q}_{h-\left[b_{k}\right], \mathcal{F}_{\mathcal{I}} \backslash \mathcal{G}_{\mathcal{I}}^{c o}}^{c}\left(\left.\left(r_{h}-b_{k}\right)\right|_{\mathcal{I}}\right)
$$

First we claim that

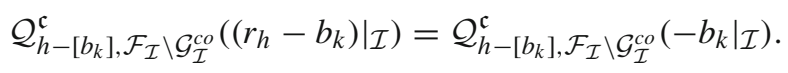

Indeed, if $\left[\sum_{i} y_{i} a_{i}\right]=h-\left[b_{k}\right]$ and $\left(\sum_{i} y_{i} a_{i}\right)_{v}<\left(r_{h}-b_{k}\right)_{v}$ for all $v \in \mathcal{I}$ (where the strictness of the inequality is imposed by the boundary condition $\mathcal{F}_{\mathcal{I}} \backslash \mathcal{G}_{\mathcal{I}}^{\text {co }}$ ) then necessarily $\left(\sum_{i} y_{i} a_{i}\right)_{v}<\left(-b_{k}\right)_{v}$ also holds for $v \in \mathcal{I}$. That is, if $[a]=h$ and $a_{v}<\left(r_{h}\right)_{v}$ then $a_{v}<0$. To see this write $a=r_{h}+l$ with $l \in L$, then $l_{v}<0$ hence $l_{v} \leq-1$, and $a_{v} \leq\left(r_{h}\right)_{v}-1<0$.

On the other hand, since $b_{k} \in \mathfrak{c}$, from (3.4.3) one has

$$
(-1)^{n} \cdot \mathcal{Q}_{-h+\left[b_{k}\right], \mathcal{G}_{\mathcal{I}}^{c o}}^{\mathfrak{c}}\left(b_{k} \mid \mathcal{I}\right)=\mathcal{Q}_{h-\left[b_{k}\right], \mathcal{F}_{\mathcal{I}}^{\mathfrak{c}} \backslash \mathcal{G}_{\mathcal{I}}^{c o}}\left(-b_{k} \mid \mathcal{I}\right)
$$

The expression $\mathcal{Q}_{-h+\left[b_{k}\right], \mathcal{G}_{\mathcal{I}}^{c o}}^{\mathfrak{c}}\left(b_{k} \mid \mathcal{I}\right)$ counts solutions of $\sum_{i} y_{i} a_{i, v} \leq b_{k, v}$ for all $v \in \mathcal{I}$ under the restrictions $\left[b_{k}-\sum_{i} y_{i} a_{i}\right]=h$ and $y_{i}>0$ for all $i$. On the other hand, in the expansion at infinity,

$$
(-1)^{n} \cdot \frac{\mathbf{t}^{b_{k}}}{\prod_{i}\left(1-\mathbf{t}^{a_{i}}\right)} \stackrel{T^{\infty}}{\longrightarrow} \mathbf{t}^{b_{k}} \cdot \sum_{y_{i}>0} \mathbf{t}^{-\sum_{i} y_{i} a_{i}} .
$$

Hence

$$
\sum_{k} \iota_{k} \cdot(-1)^{n} \cdot \mathcal{Q}_{-h+\left[b_{k}\right], \mathcal{G}_{\mathcal{I}}^{c o}}^{\mathfrak{c}}\left(\left.b_{k}\right|_{\mathcal{I}}\right)=\sum_{\tilde{l} \geq 0} r_{\mathcal{I}}^{(h)}(\tilde{l})
$$




\section{Duality for multivariable Poincaré series}

\section{1}

In this section, we will return to the situation of Sect. 2: we consider the rational function $f(\mathbf{t})$ from (2.2.1) associated with a connected negative definite plumbing graph $\Gamma$, its expansion at the origin $\mathcal{Z}(\mathbf{t})$, its expansion at infinity $T^{\infty} f(\mathbf{t})$, and their equivariant versions and reductions from Sect. 3.2. We also use all the notations from Sect. 3 regarding the counting and modified counting functions associated with $f$ and $\mathcal{Z}, \mathcal{S}_{\mathbb{R}}^{\prime}$ is the real Lipman cone, and for any $\mathcal{I}$ we consider the projection $\pi_{\mathcal{I}}: \mathbb{R}\left\langle E_{v}\right\rangle_{v \in \mathcal{V}} \rightarrow \mathbb{R}\left\langle E_{v}\right\rangle_{v \in \mathcal{I}}=\mathbb{R}^{|\mathcal{I}|}$, denoted also as $\left.x \mapsto x\right|_{\mathcal{I}}$. The projected (real) Lipman cone is the cone in $\mathbb{R}^{|\mathcal{I}|}$ defined as $\pi_{\mathcal{I}}\left(S_{\mathbb{R}}^{\prime}\right)$. Recall that in the presence of the reduction we consider the objects $\mathcal{Z}_{h}\left(\mathbf{t}_{\mathcal{I}}\right)$ for every $h \in H$, thus we keep $H=L^{\prime} / L$ as an index set and we never consider $\pi_{\mathcal{I}}\left(L^{\prime}\right) / \pi_{\mathcal{I}}(L)$, cf. Sect. 3.2.

We also review a reduction analogue of Theorem 2.4.2, which is the prototype of several results showing that for certain choices of $\mathcal{I}($ eg. $\mathcal{I}=\mathcal{N}$, where $\mathcal{N}$ denoted the set of nodes in the plumbing graph), the reduction $\mathcal{Z}_{h}\left(\mathbf{t}_{\mathcal{I}}\right)$ still preserves the 'SeibergWitten information' (for more see [21]).

Theorem 4.1.1 [20] The counting function of $\mathcal{Z}_{h}\left(\mathbf{t}_{\mathcal{N}}\right)$ in the cone $\pi_{\mathcal{N}}\left(S_{\mathbb{R}}^{\prime}\right)$ admits a quasipolynomial and a periodic constant, and

$$
\operatorname{pc}^{\pi_{\mathcal{N}}\left(S_{\mathbb{R}}^{\prime}\right)}\left(\mathcal{Z}_{h}\left(\mathbf{t}_{\mathcal{N}}\right)\right)=\mathrm{pc}^{S_{\mathbb{R}}^{\prime}}\left(\mathcal{Z}_{h}(\mathbf{t})\right)=-\mathfrak{s} \mathfrak{w}_{-h * \sigma_{c a n}}(M)-\frac{\left(K+2 r_{h}\right)^{2}+|\mathcal{V}|}{8} .
$$

Such a result has the following advantages: the number of reduced variables (i.e., in this case, the number of nodes) usually is considerably less than the number of vertices, a fact which reduces the complexity of the calculations. Moreover, the reduced series reflects more conceptually the complexity of the manifold $M$ (using only one variable for each Seifert 3-manifold piece in its JSJ-decomposition). Furthermore, the reduced series can be compared/linked with other (geometrically or analytically defined) objects as well (see e.g. [5,33]).

The above theorem has several generalizations, however the number of variables cannot be decreased arbitrarily, it is obstructed by the normalized Seiberg-Witten invariants of the complementary subgraph $\Gamma(\mathcal{V} \backslash \mathcal{I})$, for details see [21].

\section{2}

As we already mentioned, the modified counting function has some additional nice properties (compared with the original counting function). Regarding it, in the sequel we will use several results from [22] (where $q_{h, \mathcal{I}}$ associated with $f$ is called the 'coefficient function', since $q_{h, \mathcal{I}}\left(l_{0}^{\prime}\right)$ is the coefficient of $\mathbf{t}_{\mathcal{I}}^{l_{0}^{\prime}}$ in the Taylor expansion of $\left.f_{h}\left(\mathbf{t}_{\mathcal{I}}\right) \cdot \prod_{v \in \mathcal{I}} \mathbf{t}_{\mathcal{I}}^{E_{v}} /\left(1-\mathbf{t}_{\mathcal{I}}^{E_{v}}\right)\right)$. 


\section{3 'Chamber property'}

If we wish to apply Theorem 3.6.1 for $q_{h, \mathcal{I}}$, we need to find a chamber associated with the denominator of the rational function $f\left(\mathbf{t}_{\mathcal{I}}\right)$, which contains all vectors of type $\left.b_{k}\right|_{\mathcal{I}}$ where $b_{k}$ are the exponents appearing in the numerator. The next proposition shows the existence of a chamber which contains the whole projected real Lipman cone. In order to give some intuition for this fact, we also provide an intermediate step of its proof.

For a subset $\mathcal{I} \subset \mathcal{V}, \mathcal{I} \neq \varnothing$ we define its closure $\overline{\mathcal{I}}$ as the set of vertices of that connected minimal full subgraph $\Gamma_{\overline{\mathcal{I}}}$ of $\Gamma$, which contains $\mathcal{I}$. We denote by $\delta_{v, \overline{\mathcal{I}}}$ the valency of a vertex $v \in \overline{\mathcal{I}}$ in the graph $\Gamma_{\overline{\mathcal{I}}}$.

In [22, Lemma 11] is proved that $f\left(\mathbf{t}_{\mathcal{I}}\right)$ has a product decomposition of type

$$
f\left(\mathbf{t}_{\mathcal{I}}\right)=R\left(\mathbf{t}_{\mathcal{I}}\right) \cdot \prod_{v \in \overline{\mathcal{I}}}\left(1-\mathbf{t}_{\mathcal{I}}^{E_{v}^{*}}\right)^{\delta_{v, \overline{\mathcal{I}}}-2}
$$

where $R$ is a polynomial supported on $\pi_{\mathcal{I}}\left(\mathcal{S}^{\prime}\right)$, in particular it has no pole. Hence, the possible poles of $f$ via the $\mathcal{I}$-reduction are replaced from the set of poles of $\left\{1-\mathbf{t}^{E_{v}^{*}}\right\}_{v \in \mathcal{E}}$ to the set of poles of $\left\{1-\mathbf{t}_{\mathcal{I}}^{E_{v}^{*}}\right\}_{v \in \mathcal{E}_{\overline{\mathcal{I}}}}$. Here $\mathcal{E}_{\overline{\mathcal{I}}}$ is the set of end-vertices of $\Gamma_{\overline{\mathcal{I}}}$; note that $\mathcal{E}_{\overline{\mathcal{I}}} \subset \mathcal{I}$. Therefore, by the construction of the chamber decomposition of $\mathbb{R}^{|\mathcal{I}|}$ (cf. 3.4) the chambers associated with $f\left(\mathbf{t}_{\mathcal{I}}\right)$ are determined by the bases selected from $\left\{\left.E_{v}^{*}\right|_{\mathcal{I}},\left.E_{u}\right|_{\mathcal{I}}: v \in \mathcal{E}\left(\Gamma_{\overline{\mathcal{I}}}\right), u \in \mathcal{I}\right\}$. These facts and a lattice-combinatorial argument provide

Proposition 4.3.2 [22] For any $\mathcal{I}$ the interior of the projected Lipman cone int $\left(\pi_{\mathcal{I}}\left(\mathcal{S}_{\mathbb{R}}^{\prime}\right)\right.$ ) is contained entirely in a (big) chamber $\mathfrak{c}$ of $f\left(\mathbf{t}_{\mathcal{I}}\right)$.

Remark 4.3.3 In [21,22] is also proved the following 'convexity property': If $l_{0}^{\prime} \in$ $Z_{K}-E+\mathcal{S}^{\prime}$ and $\left[l_{0}^{\prime}\right]=h$ then $q_{h, \mathcal{I}}\left(l_{0}^{\prime}\right)=q_{h, \overline{\mathcal{I}}}\left(l_{0}^{\prime}\right)$.

\subsection{Duality for counting functions and periodic constants}

Now we are ready to prove our main theorem: a duality/pairing between special evaluations of the counting functions associated with $f$ and the periodic constants. The duality is the upshot of two 'symmetries', manifested at two different levels. The first one is the equivariant Ehrhart-Macdonald-Stanley reciprocity of the polytopes, while the second is a topological imprint of the Gorenstein duality present at the level of the topological Poincaré series: a $\left\{x \leftrightarrow Z_{K}-x\right\}$ symmetry.

Theorem 4.4.1 Fix any $\mathcal{I} \subset \mathcal{V}, \mathcal{I} \neq \emptyset$ and $h \in H$. Then

(a) $\operatorname{mpc}^{\pi \mathcal{I}\left(\mathcal{S}_{\mathbb{R}}^{\prime}\right)}\left(\mathcal{Z}_{h}\left(\mathbf{t}_{\mathcal{I}}\right)\right)=q_{\left[Z_{K}\right]-h, \mathcal{I}}\left(Z_{K}-r_{h}\right)$;

(b) $\mathrm{pc}^{\pi_{\mathcal{I}}\left(\mathcal{S}_{\mathbb{R}}^{\prime}\right)}\left(\mathcal{Z}_{h}\left(\mathbf{t}_{\mathcal{I}}\right)\right)=Q_{\left[Z_{K}\right]-h, \mathcal{I}}\left(Z_{K}-r_{h}\right)$.

That is, (in principle, the hardly computable) periodic constant of the series $\mathcal{Z}_{h}$ can be determined as a precise finite sum of coefficients of the dual series $\mathcal{Z}_{\left[Z_{K}\right]-h}$. 
Proof By the inclusion-exclusion principle (3.3.3) $(b)$ is implied by $(a)$. Next we prove $(a)$.

The substitution $x \mapsto Z_{K}-x$ in $f$, together with the identities $Z_{K}-E=$ $\sum_{v \in \mathcal{V}}\left(\delta_{v}-2\right) E_{v}^{*}$ from (2.1.1) and $-2=\sum_{n \in \mathcal{V}}\left(\delta_{n}-2\right)$ (since $\Gamma$ is a tree) gives

$$
f\left(\mathbf{t}_{\mathcal{I}}\right)=\mathbf{t}_{\mathcal{I}}^{Z_{K}-E} \cdot f\left(\mathbf{t}_{\mathcal{I}}^{-1}\right)
$$

This on the Taylor expansion level transforms into the symmetry $T^{\infty} f\left(\mathbf{t}_{\mathcal{I}}\right)=$ $\mathbf{t}_{\mathcal{I}}^{Z_{K}-E} \mathcal{Z}\left(\mathbf{t}_{\mathcal{I}}^{-1}\right)=\sum_{l^{\prime} \in \mathcal{S}^{\prime}} z\left(l^{\prime}\right) \mathbf{t}_{\mathcal{I}}^{Z_{K}-E-l^{\prime}}$. The corresponding $h$-equivariant parts are

$$
\left(T^{\infty} f\right)_{h}\left(\mathbf{t}_{\mathcal{I}}\right)=\sum_{l^{\prime} \in \mathcal{S}^{\prime},\left[l^{\prime}\right]=\left[Z_{K}\right]-h} z\left(l^{\prime}\right) \mathbf{t}_{\mathcal{I}}^{Z_{K}-E-l^{\prime}} .
$$

Furthermore, (4.3.1) and the sentence after it shows that in the numerator of $f\left(\mathbf{t}_{\mathcal{I}}\right)$ all the exponents are situated in the projected Lipman cone $\pi_{\mathcal{I}}\left(\mathcal{S}_{\mathbb{R}}^{\prime}\right)$. In particular, by Lemma 4.3.2, they are contained in a fixed chamber $\mathfrak{c} \subset \mathbb{R}^{|\mathcal{I}|}$ which contains $\pi_{\mathcal{I}}\left(\mathcal{S}_{\mathbb{R}}^{\prime}\right)$. Therefore Theorem 3.6.1 gives

$$
\operatorname{mpc}^{\pi_{\mathcal{I}}\left(\mathcal{S}_{\mathbb{R}}^{\prime}\right)}\left(\mathcal{Z}_{h}\left(\mathbf{t}_{\mathcal{I}}\right)\right)=\sum_{\left.\left.l^{\prime}\right|_{\mathcal{I} \leq}\left(Z_{K}-E\right)\right|_{\mathcal{I}},\left[l^{\prime}\right]=\left[Z_{K}\right]-h} z\left(l^{\prime}\right)
$$

But the right-hand side is exactly the counting function $q_{\left[Z_{K}\right]-h, \mathcal{I}}\left(Z_{K}-r_{h}\right)$, since $\left.l^{\prime}\right|_{\mathcal{I}} \leq\left.\left(Z_{K}-E\right)\right|_{\mathcal{I}}$ is equivalent with $\left.\left.l^{\prime}\right|_{\mathcal{I}} \prec\left(Z_{K}-r_{h}\right)\right|_{\mathcal{I}}$ if $\left[l^{\prime}\right]=\left[Z_{K}\right]-h$.

Corollary 4.4.4 $Q_{\left[Z_{K}\right]-h, \mathcal{N}}\left(Z_{K}-r_{h}\right)=-\mathfrak{s w}_{h}^{\text {norm }}(M)$; that is, the Seiberg-Witten invariant can be expressed via the counting function as a finite sum of $\mathcal{Z}$-coefficients.

Proof Combine Theorems 4.1.1 and 4.4.1.

\section{The 'polynomial part' of the series $\mathcal{Z}(t)$}

\subsection{The 'Polynomial-negative degree part' decomposition: motivation and history}

Consider a one-variable rational function $z(t)=B(t) / A(t)$ with $A(t)=\prod_{i=1}^{n}\left(1-t^{a_{i}}\right)$ and $a_{i}>0$. In [5, 7.0.2] is observed that any such function has a unique decomposition of the form $z(t)=P^{+}(t)+z^{n e g}(t)$, where $P^{+}(t)$ is a polynomial (with non-negative exponents) and $z^{n e g}(t)$ is a rational function of negative degree. Furthermore, $z(t)$ admits a periodic constant (associated with the Taylor expansion of $z$ and the cone $\mathbb{R}_{\geq 0}$ ), which equals $P^{+}(1)$. The decomposition can be established using the Euclidean division algorithm. $P^{+}$is called the polynomial part while the rational function $z^{\text {neg }}$ is the negative degree part of the decomposition.

This one-variable periodic constant computation was useful in theorems of type 4.1.1 with only one node (Seifert 3-manifolds), or surgery formulas along one node, cf. [5]. Basically, in these cases, the concrete computation of the periodic constant was based on the computation of $P^{+}$. 
Though the multivariable generalization was hardly needed, till now the complete answer was not found. The main questions were: (a) what are the universal properties of the parts $P^{+}$and $z^{n e g}$, which guarantee that a decomposition $z=P^{+}+z^{n e g}$ exists and it is unique; and, (b) what algorithm provides this decomposition. Additionally, the wished decomposition must satisfy (at least) the next basic property: (c) in the geometric context $z(\mathbf{t})=f(\mathbf{t})$, cf. (2.2.1), pc $(z)=P^{+}(1)$, where pc is associated with the Lipman cone (or, at least, with some subcone of it). (Hence, in this geometric situation, when all these are satisfied, whenever the Seiberg-Witten invariant is computable via such periodic constant, e.g. when $\mathcal{I}$ contains all the nodes, cf. Theorem 4.1.1, or, the 'bad' vertices of $\Gamma$, cf. [21], then $P^{+}$is a polynomial generalization of the Seiberg-Witten invariant.)

For functions with two variables in [20] a decomposition was established satisfying all the required properties, based on a 'two-variable division procedure'. Furthermore, for more variables, [22] constructed a candidate polynomial $P_{1}^{+}$based on the combinatorics of $\Gamma$, Ehrhart theory and reduction to the one- and two-variable divisions. It satisfied (c), but it didn't answer (a) in a natural way. Later, [23] considered another polynomial $P_{2}^{+}$(with $P_{2}^{+} \neq P_{1}^{+}$in general) constructed via an inductive multivariable Euclidean division. It answered (a)-(b), but (c) was not established, so it was not clear if $P_{2}^{+}$is helpful at all in pc (or Seiberg-Witten) computations. The authors of [23, 4.4] not quite conjectured, but asked convincingly whether this $P_{2}^{+}$is the right candidate for the polynomial part. Below we show that this is indeed the case.

\subsection{The polynomial part by division [23]}

We review in short the needed statement of [23] reorganized from the point of view of the present note. We start, similarly as in Sect. 3, with a pair of free $\mathbb{Z}$-modules $L \subset L^{\prime}$, and a general multivariable rational function (in variables $\mathbf{t}^{L^{\prime}}$ )

$$
z(\mathbf{t})=\frac{\sum_{k=1}^{r} \iota_{k} \mathbf{t}^{b_{k}}}{\prod_{i=1}^{n}\left(1-\mathbf{t}^{a_{i}}\right)},
$$

where $\left\{\iota_{k}\right\}_{k=1}^{r} \in \mathbb{Z},\left\{b_{k}\right\}_{k=1}^{r},\left\{a_{i}\right\}_{i=1}^{n} \in L^{\prime}$ such that $b_{k} \nprec 0$ for all $k$ and $0 \prec a_{i}$ for all $i$. (In our application $z$ will be $f_{h}\left(\mathbf{t}_{\mathcal{I}}\right)$ for some $\mathcal{I} \neq \emptyset$.)

\section{Proposition 5.2.1 [23]}

(a) $z(\mathbf{t})$ can be written in the following form by a 'multivariable Euclidean division algorithm':

$$
z(\mathbf{t})=\sum_{S \subset\{1, \ldots, n\}} \frac{\sum_{j} \iota_{S, j} \mathbf{t}^{b_{S, j}}}{\prod_{i \in S}\left(1-\mathbf{t}^{a_{i}}\right)},
$$

where $\iota_{S, j} \in \mathbb{Z}, b_{S, j} \nprec 0$ for all $(S, j)$, and $b_{S, j} \prec a_{i}$ for all $(S, j)$ and all $i \in S$ whenever $\mathcal{S} \neq \emptyset$. $(b) z(\mathbf{t})$ has a decomposition of type $P^{+}(\mathbf{t})+z^{\text {neg }}(\mathbf{t})$ with the next properties:

(i) $P^{+}(\mathbf{t})$ is a finite sum (polynomial) $\sum_{j} n_{j} \mathbf{t}^{c_{j}}$ with $c_{j} \nprec 0$ for all $j$;

(ii) $z^{\text {neg }}(\mathbf{t})$ is a rational function with negative degree in all variables $t_{i}$. 
Furthermore, a decomposition of $z(\mathbf{t})$ with properties $(i)$-(ii) is unique.

(c) In fact, the terms $P^{+}$and $z^{\text {neg }}$ of the decomposition from (b) are given via (5.2.2) as follows: $P^{+}$(resp. $z^{\text {neg }}$ ) is the sum of terms from (5.2.2) over $S=\emptyset$ (resp. $S \neq \varnothing)$.

Proof (a) Assume that we have an expression $g(\mathbf{t})=\mathbf{t}^{b} / \prod_{i \in S}\left(1-\mathbf{t}^{a_{i}}\right)$ such that $b \nprec 0$ and there exists some $i_{0}$ such that $b \nprec a_{i_{0}}$. Then we replace $g$ by $-\mathbf{t}^{b-a_{i_{0}}} / \prod_{i \in S \backslash\left\{i_{0}\right\}}\left(1-\mathbf{t}^{a_{i}}\right)+\mathbf{t}^{b-a_{i_{0}}} / \prod_{i \in S}\left(1-\mathbf{t}^{a_{i}}\right)$. Note that $b-a_{i_{0}} \nprec 0$, hence the new fractions have similar form. Starting from the original expression of $z$, by repeating the above step whenever is applicable, after finitely many steps we obtain (5.2.2).

(b) Define $P^{+}$and $z^{n e g}$ as is indicated in (c). Then properties ( $i$ )-(ii) are automatically satisfied. Next, we prove the uniqueness of the decomposition. We need to show that if $P^{+}(\mathbf{t})+z^{n e g}(\mathbf{t})=0(\dagger)$ and if $P^{+}$and $z^{\text {neq }}$ satisfy $(i)-(i i)$, then both are zero. But, from $(\dagger)$, both $P^{+}$and $z^{\text {neq }}$ should be simultaneously polynomials and also rational functions of negative degrees in all the variables, a fact which happens only of they are zero since a rational function can be represented as a rational function in a unique way.

\subsection{The polynomial part of $f_{h}\left(t_{\mathcal{I}}\right)$ by duality}

Assume again that we are in the situation of a plumbing graph and its rational function $f(\mathbf{t})$ as in Sects. 2 and 4 .

We fix $h \in H$. Then, by the proof of Theorem 4.4.1 we have $f_{h}\left(\mathbf{t}_{\mathcal{I}}\right)=$ $\mathbf{t}_{\mathcal{I}}^{Z_{K}-E} \cdot f_{\left[Z_{K}\right]-h}\left(\mathbf{t}_{\mathcal{I}}^{-1}\right)$ and $T^{\infty} f_{h}\left(\mathbf{t}_{\mathcal{I}}\right)=\mathbf{t}_{\mathcal{I}}^{Z_{K}-E} \mathcal{Z}_{\left[Z_{K}\right]-h}\left(\mathbf{t}_{\mathcal{I}}^{-1}\right)$. Write this expression $\mathbf{t}_{\mathcal{I}}^{Z_{K}-E} \mathcal{Z}_{\left[Z_{K}\right]-h}\left(\mathbf{t}_{\mathcal{I}}^{-1}\right)$ as $\sum_{l^{\prime} \in Z_{K}-E-\mathcal{S}^{\prime}} w\left(l^{\prime}\right) \mathbf{t}_{\mathcal{I}}^{l^{\prime}}$, where $\left[l^{\prime}\right]=h$ automatically whenever $w\left(l^{\prime}\right) \neq 0$. Define

$$
P_{h, \mathcal{I}}^{+}\left(\mathbf{t}_{\mathcal{I}}\right):=\sum_{l^{\prime}|\mathcal{I} \nprec 0|_{\mathcal{I}}} w\left(l^{\prime}\right) \mathbf{t}_{\mathcal{I}}^{l^{\prime}}, \quad f_{h, \mathcal{I}}^{n e g}\left(\mathbf{t}_{\mathcal{I}}\right):=\sum_{l^{\prime}|\mathcal{I} \prec 0|_{\mathcal{I}}} w\left(l^{\prime}\right) \mathbf{t}_{\mathcal{I}}^{l^{\prime}} .
$$

Write also $P_{h, \mathcal{I}}^{+}(\mathbf{1}):=\left.P_{h, \mathcal{I}}^{+}\left(\mathbf{t}_{\mathcal{I}}\right)\right|_{t_{i}=1, \forall i}$.

Theorem 5.3.2 Consider the decomposition $f_{h}\left(\mathbf{t}_{\mathcal{I}}\right)=P_{h, \mathcal{I}}^{+}\left(\mathbf{t}_{\mathcal{I}}\right)+f_{h, \mathcal{I}}^{\text {neg }}\left(\mathbf{t}_{\mathcal{I}}\right)$ from (5.3.1).

(a) $P_{h, \mathcal{I}}^{+}\left(\mathbf{t}_{\mathcal{I}}\right)$ and $f_{h, \mathcal{I}}^{n e g}\left(\mathbf{t}_{\mathcal{I}}\right)$ satisfy the requirements $(i)$-(ii) from Proposition 5.2.1(b). In particular, by the uniqueness of the decomposition, this decomposition agrees with the decomposition from 5.2.1(a)-(c) given by Euclidean division.

(b) $P_{h, \mathcal{I}}^{+}(\mathbf{1})=Q_{\left[Z_{K}\right]-h, \mathcal{I}}\left(Z_{K}-r_{h}\right)$. In particular, $P_{h, \mathcal{I}}^{+}(\mathbf{1})=\operatorname{pc}^{\pi_{\mathcal{I}}\left(\mathcal{S}_{\mathbb{R}}^{\prime}\right)}\left(\mathcal{Z}_{h}\left(\mathbf{t}_{\mathcal{I}}\right)\right)$.

Proof (a) By its definition, $P_{h, \mathcal{I}}^{+}\left(\mathbf{t}_{\mathcal{I}}\right)$ is a finite sum, hence $f_{h, \mathcal{I}}^{n e g}\left(\mathbf{t}_{\mathcal{I}}\right)=f_{h}\left(\mathbf{t}_{\mathcal{I}}\right)-$ $P_{h, \mathcal{I}}^{+}\left(\mathbf{t}_{\mathcal{I}}\right)$ is a rational function. Since in its expansion all monomials $w\left(l^{\prime}\right) \mathbf{t}_{\mathcal{I}}^{l^{\prime}}$ satisfy $\left.\left.l^{\prime}\right|_{\mathcal{I}} \prec 0\right|_{\mathcal{I}}$, it has negative degree in all the variables. 
(b) By the above transformations $w\left(l^{\prime}\right)=z\left(Z_{K}-E-l^{\prime}\right),\left[l^{\prime}\right]=h$. Hence $\left.\left.l^{\prime}\right|_{\mathcal{I}} \nprec 0\right|_{\mathcal{I}}$ transforms into $\left.\left.\left(Z_{K}-E\right)\right|_{\mathcal{I}} \nprec\left(Z_{K}-E-l^{\prime}\right)\right|_{\mathcal{I}}$, or $\left.\left.\left(Z_{K}-E-l^{\prime}\right)\right|_{\mathcal{I}} \ngtr\left(Z_{K}-r_{h}\right)\right|_{\mathcal{I}}$. This proves the first identity. For the second one use Theorem 4.4.1 (b).

Corollary 4.4.4 and Theorem 5.3.2 combined gives

Corollary 5.3.3 For any $h \in H$ one has $P_{h, \mathcal{N}}^{+}(\mathbf{1})=-\mathfrak{s w}_{h}^{\text {norm }}(M)$.

We illustrate the above duality results on the following example.

Example 5.3.4 Let us consider the Brieskorn sphere $M=\Sigma(2,5,7)$. Recall that the entries of $E_{v}^{*}$ 's are the corresponding columns of the matrix $\left\{-\left(E_{i}^{*}, E_{j}^{*}\right)\right\}_{i, j}$, which is in fact $-I^{-1}$, the negative of the inverse of the intersection matrix. In this case the graph and $-I^{-1}$ are the following:

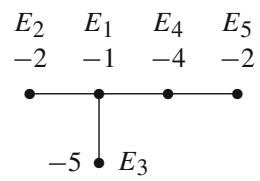

$$
-I^{-1}=\left[\begin{array}{ccccc}
70 & 35 & 14 & 20 & 10 \\
35 & 18 & 7 & 10 & 5 \\
14 & 7 & 3 & 4 & 2 \\
20 & 10 & 4 & 6 & 3 \\
10 & 5 & 2 & 3 & 2
\end{array}\right]
$$

Therefore, the zeta-function reduced to the set of nodes $\mathcal{N}=\left\{v_{0}\right\}$ and its 'polynomial-negative degree part' decomposition (obtained by simple division of polynomials) can be written as

$$
f(t)=\frac{1-t^{70}}{\left(1-t^{35}\right)\left(1-t^{14}\right)\left(1-t^{10}\right)}=t+t^{11}+\frac{1-t+t^{15}+t^{21}}{\left(1-t^{14}\right)\left(1-t^{10}\right)} .
$$

The same result follows by duality as well. Indeed, the Taylor expansion is $\mathcal{Z}(t)=$ $1+t^{10}+t^{14}+\cdots$ and one calculates $Z_{K}=(12,6,3,4,2)$ where we use short notation $\left(l_{1}, l_{2}, l_{3}, l_{4}, l_{5}\right)$ for $l=\sum_{i=1}^{5} l_{i} E_{i}$. Hence, for $t^{Z_{K}-E} \mathcal{Z}\left(t^{-1}\right)$ (where $t^{Z_{K}-E}:=t^{\left(Z_{K}\right)_{0}{ }^{-1}}$, see the notation from 3.2) we get $t^{11}+t^{1}+t^{-3}+\cdots$, which gives $P^{+}(t)=t^{11}+t$ again.

\subsection{Example: plane curve singularities}

Let us describe the analogue of (3.6.2) and of the 'polynomial-negative degree part' decomposition for the embedded situation of an irreducible plane curve singularity $g:\left(\mathbb{C}^{2}, 0\right) \rightarrow(\mathbb{C}, 0)$. In this case the series $z(t)$ is the Taylor expansion at the origin of the monodromy zeta function $\zeta(t)=\Delta(t) /(1-t)$, cf. [1,2]. A'Campo's formula constructs $\zeta(t)$ via (2.2.1), but now applied to the minimal embedded resolution graph $\Gamma_{g}$ of the plane germ (see Fig. 2), whose unique (-1)-vertex has an extra arrowheadneighbour (where the arrow represents the strict transform of $\{g=0\}$, thus the $(-1)$ vertex is considered to be a node of the graph), and this series is reduced to the variable of the $(-1)$-node. (Below the doted lines represent string subgraphs.)

Let $\mathcal{M} \subset \mathbb{Z}_{\geq 0}$ be the semigroup of $g$ consisting of all intersection multiplicities of $g$ with all possible analytic germs. By [7] $T \zeta(t)=\sum_{s \in \mathcal{M}} t^{s}$. Let $\mu=\operatorname{deg}(\Delta)$ be the Milnor number of $g$. We determine the polynomial part of $T \zeta(t)$ and we verify the 
Fig. 2 The shape of the minimal embedded resolution graph of $g$

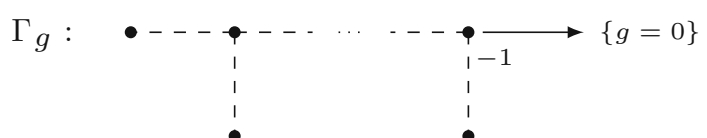

analogue of (3.6.2). Here the needed Gorenstein duality reads as follows: $(\dagger) s \notin \mathcal{M}$ if and only if $\mu-1-s \in \mathcal{M}$. This also shows that $\mathbb{Z} \backslash \mathcal{M}$ is finite, its cardinality is exactly $\mu / 2$, and the largest element of $\mathbb{Z} \backslash \mathcal{M}$ is $\mu-1$.

Since $T \zeta(t)=\sum_{s \in \mathcal{M}} t^{s}=\sum_{s \geq 0} t^{s}-\sum_{s \notin \mathcal{M}, s \geq 0} t^{s}$ we get that $\zeta^{n e g}(t)=$ $\sum_{s \geq 0} t^{s}=1 /(1-t)$, and $P^{+}(t)=-\sum_{s \notin \mathcal{M}, s \geq 0} t^{s}$. In particular, $\operatorname{pc}(\zeta)=P^{+}(1)=$ $-\mu / 2$. On the other hand, by duality $(\dagger)$ one has $T^{\infty} \zeta(t)=-t^{\mu-1} \cdot T \zeta\left(t^{-1}\right)=$ $-t^{\mu-1}\left(\sum_{s \in \mathcal{M}, s<\mu} t^{-s}+\sum_{s \geq \mu} t^{-s}\right) \stackrel{(\dagger)}{=}-\sum_{s \notin \mathcal{M}, s \geq 0} t^{s}-\left(t^{-1}+t^{-2}+\cdots\right)=$ $P^{+}(t)-\left(t^{-1}+t^{-2}+\cdots\right)$, whose part with positive exponents is exactly $P^{+}(t)$.

[In this 'relative case', the geometric object is not a 3-manifold, but a knot in $S^{3}$. By the above computation, the periodic constant of the corresponding 'relative' series is $-\mu / 2$, where $\mu$ serves as the genus of the knot as well. $\mu / 2$ is in fact the 'delta-invariant' of the algebraic knot, which is the low-dimensional analogue of the 'geometric genus' defined for surfaces.]

\section{Topological polytopes, counting lattice points and Seiberg-Witten invariants}

\subsection{Motivation: polytopes and invariants of normal surface singularities}

Results which connect geometric and topological invariants of algebraic varieties with the number of lattice points and the volume of certain polytopes has a long history and it culminates in toric geometry. At the level of singularities, the very first class where this phenomenon appears is the Newton non-degenerate hypersurface singularities (see eg. [2]). In this case one defines the Newton polytope $\mathcal{P}$ associated with the defining equation and one proves that several invariants of the singularity can be recovered from $\mathcal{P}$, see the following non-complete list of articles: [6,11,19,27,46,49]. In the present article we would like to highlight only the geometric genus $p_{g}$, which equals the number of lattice points with strictly positive coordinates in $\mathcal{P}$, cf. [27]. Moreover, [46] shows that in the case when the germ has a rational homology sphere link $M$, one has $p_{g}=-\mathfrak{s w}_{0}^{\text {norm }}(M)$ supporting the Seiberg-Witten invariant conjecture of Némethi and Nicolaescu [36] for this case and, in particular, expressing the SeibergWitten invariant as a lattice point counting in the Newton polytope.

The analytic part can be generalized to the case of isolated complete intersection singularities with certain non-degeneracy condition, cf. [4,18,29,42,43]. For such germs, $p_{g}$ is expressed by $[18,29]$ as an alternating sum of lattice points in certain polytopes obtained as Minkowski sums of Newton polyhedra defined by the equations of the germ.

Having in mind the above correspondence $p_{g}=-\mathfrak{s w}_{0}^{\text {norm }}(M)$ between the geometric genus and the Seiberg-Witten invariant (formulated by 'Seiberg-Witten invariant 
conjecture' and valid for certain analytic types of singularities), it is natural to ask if there is an analogous interpretation of the Seiberg-Witten invariant in terms of an alternating sum of lattice points in certain polytopes associated with the topological type (valid in any topological situation). We will call such expression inclusion-exclusion lattice point counting, or IELP counting.

The main result (and novelty) of this section is a positive answer to this question, valid for any normal surface singularity with rational homology sphere link. Though in the case of $p_{g}$ and Merle-Teissier-Khovanskii-Morales type formulas the polytopes were associated with the equation of the germ, in this topological version they are associated with the resolution graph.

The wished expression starts with the results from [20], which identifies the Seiberg-Witten invariant with certain coefficients of the Ehrhart quasipolynomial of a dilated polytope associated with the plumbing graph of the manifold $M$. However, merely with the techniques of [20] a concrete lattice point counting was obstructed. The point is that the duality and construction of $P^{+}$in the present note unlock the obstructions of [20] in the direction of an IELP counting.

\subsection{Preparation for an IELP expression}

In Sect. 3.4 we have defined the linear map $\mathfrak{l}: \mathbb{R}^{|\mathcal{E}|} \rightarrow \mathbb{R}^{|\mathcal{V}|}$ by $\mathfrak{l}(\mathbf{y})=\sum_{e \in \mathcal{E}} y_{e} E_{e}^{*}$ for any $\mathbf{y}=\left(y_{e}\right)_{e \in \mathcal{E}} \in \mathbb{R}^{|\mathcal{E}|}$ (where $\mathcal{E}$ is the set of end-vertices, and in (2.2.1) $\prod_{e \in \mathcal{E}}(1-$ $\mathbf{t}^{E_{e}^{*}}$ ) is the denominator of $f$ ). Additionally, we have considered also the representation given by the composition $\rho: \mathbb{Z}^{|\mathcal{E}|} \stackrel{\mathfrak{l}_{\mathbb{Z}}|\mathcal{E}|}{\longrightarrow} L^{\prime} \longrightarrow H=L^{\prime} / L$. Set also the notation $\mathfrak{l}_{\mathcal{I}}$ for the map defined by the composition of $\mathfrak{l}$ with the projection $\pi_{\mathcal{I}}: \mathbb{R}^{|\mathcal{V}|} \rightarrow \mathbb{R}^{|\mathcal{I}|}$. In particular, for $\mathcal{I}=\{v\}$ the maps $\mathfrak{l}_{v}$ are the components of $\mathfrak{l}$.

Recall also that for any $\mathcal{I} \subset \mathcal{V}, \mathcal{I} \neq \emptyset$ we have defined the closed dilated convex polytopes $\mathcal{P}_{\mathcal{I}}^{\left(l^{\prime}\right)}$ indexed by $l^{\prime} \in L^{\prime}: \mathcal{P}_{\mathcal{I}}^{\left(l^{\prime}\right)}=\cap_{v \in \mathcal{I}} \mathcal{P}_{v}^{\left(l^{\prime}\right)}$, where $\mathcal{P}_{v}^{\left(l^{\prime}\right)}=\left\{\mathbf{y} \in \mathbb{R}_{\geq 0}^{|\mathcal{E}|}\right.$ : $\left.\left.\sum_{e \in \mathcal{E}} y_{e} E_{e}^{*}\right|_{v} \leq l_{v}^{\prime}\right\}$

In this section (similarly as in [20]) we consider also the dilated concave polytopes as well:

$$
\widetilde{\mathcal{P}}_{\mathcal{I}}^{\left(l^{\prime}\right)}=\bigcup_{v \in \mathcal{I}} \mathcal{P}_{v}^{\left(l^{\prime}\right)} \subset \mathbb{R}_{\geq 0}^{|\mathcal{E}|} .
$$

From the definition it is clear that $\widetilde{\mathcal{P}}_{\mathcal{I}}^{\left(l^{\prime}\right)}$ depends only on the restriction $\left.l^{\prime}\right|_{\mathcal{I}}$. Furthermore, it can happen that in the union the contribution of some $\mathcal{P}_{v}^{\left(l^{\prime}\right)}$ is superfluous. This is what we clarify next.

If for some $\mathcal{I} \subset \mathcal{V}$ one has $l^{\prime}=\sum_{v \in \mathcal{I}} a_{v} E_{v}^{*}$ with all $a_{v} \neq 0$, then we say that the $E^{*}$-support of $l^{\prime}$ is $\mathcal{I}$, and we write $s p^{*}\left(l^{\prime}\right)=\mathcal{I}$. Though in the sequel we are interested mainly in those cases when the $E^{*}$-support of $l^{\prime}$ is contained in $\mathcal{N}$, we formulate the following 'inclusion property' of the polytopes $\widetilde{\mathcal{P}}_{\mathcal{I}}^{\left(l^{\prime}\right)}$ for an arbitrary $\mathcal{I}$.

Lemma 6.2.2 Assume that $s p^{*}\left(l^{\prime}\right)=\mathcal{I} \neq \emptyset$. Then $\widetilde{\mathcal{P}}_{v}^{\left(l^{\prime}\right)} \subset \widetilde{\mathcal{P}}_{\mathcal{I}}^{\left(l^{\prime}\right)}$ for any $v \in \mathcal{V}$.

Proof The $\mathcal{I}=\mathcal{N}$ case appears in the proof of Theorem 5.4.2(a) of [20]. The general case is rather similar, for the convenience of the reader we sketch the proof. We assume 
that $v \notin \mathcal{I}$. Let $\Gamma_{1}$ be that connected component of the graph $\Gamma \backslash \mathcal{I}$ which contains $v$. Then consider those vertices $\left\{u_{k}\right\}_{k \in \mathcal{I}^{\prime}}$ of $\mathcal{I}$, which are adjacent to $\Gamma_{1}\left(\mathcal{I}^{\prime} \subset \mathcal{I}\right.$, $\left|\mathcal{I}^{\prime}\right|>0$ ). We claim that there exists positive rational numbers $\left\{r_{k}\right\}_{k \in \mathcal{I}^{\prime}}$ such that

$$
E_{v}^{*}-\sum_{k} r_{k} \cdot E_{u_{k}}^{*}=i\left(E_{v}^{*}\left(\Gamma_{1}\right)\right)
$$

where $i$ is the natural inclusion $L\left(\Gamma_{1}\right) \hookrightarrow L(\Gamma)$. The numbers $r_{k}$ can be determined if we intersect the identity (6.2.3) with $E_{u_{k}}$, that is, $r_{k}=\left(i\left(E_{v}^{*}\left(\Gamma_{i}\right)\right), E_{u_{k}}\right)$. Once the numbers $r_{k}$ are determined in such a way, one verifies the identity (6.2.3) by intersecting both sides with every $E_{v}$.

(6.2.3) shows that $(\dagger) E_{v}^{*}=\sum_{k} r_{k} E_{u_{k}}^{*}+\sum_{u \in \mathcal{V}\left(\Gamma_{1}\right)} r_{u} E_{u}$ for certain positive

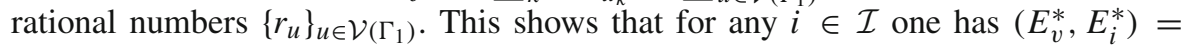
$\sum_{k} r_{k}\left(E_{u_{k}}^{*}, E_{i}^{*}\right)$, hence by the support assumption $\left(E_{v}^{*}, l^{\prime}\right)=\sum_{k} r_{k}\left(E_{u_{k}}^{*}, l^{\prime}\right)$, or $l_{v}^{\prime}=\sum_{k} r_{k} l_{u_{k}}^{\prime}$. Furthermore, the intersection of $(\dagger)$ with $E_{e}^{*}$ for $e \in \mathcal{E}$ gives $\left.E_{e}^{*}\right|_{v} \geq\left.\sum_{k} r_{k} E_{e}^{*}\right|_{u_{k}}$. These facts prove that $\widetilde{\mathcal{P}}_{v}^{\left(l^{\prime}\right)} \subset \cup_{k} \widetilde{\mathcal{P}}_{u_{k}}^{\left(l^{\prime}\right)}$.

The duality results of the present article motivate us to distinguish a special element from $L^{\prime}$

$$
l_{\text {top }}^{\prime}:=Z_{K}-E+\sum_{v \in \mathcal{E}} E_{v}^{*}
$$

which defines the special polytope $\widetilde{\mathcal{P}}_{\mathcal{V}}^{\left(l_{\text {top }}^{\prime}\right)}$. By (2.1.1) $l_{\text {top }}^{\prime}=\sum_{v \in \mathcal{N}}\left(\delta_{v}-2\right) E_{v}^{*}$ too, hence $s p^{*}\left(l_{\text {top }}^{\prime}\right)=\mathcal{N}$ and by Lemma 6.2.2 we obtain $\widetilde{\mathcal{P}}_{\mathcal{V}}^{\left(l_{\text {top }}^{\prime}\right)}=\widetilde{\mathcal{P}}_{\mathcal{N}}^{\left(l_{\text {top }}^{\prime}\right)}$. We call it the topological polytope associated with the graph $\Gamma$ and we denote it by $\widetilde{\mathcal{P}}_{\mathcal{N}}^{\text {top }}$.

A series of sub-polytopes of $\widetilde{\mathcal{P}}_{\mathcal{N}}^{\left(l_{\text {top }}^{\prime}\right)}$ are defined as follows. Let us write the set of nodes as $\mathcal{N}=\left\{v_{1}, \ldots, v_{s}\right\}$. Then consider the multiset $\mathcal{N}^{m}:=\left\{v_{1}^{\delta_{v_{1}}-2}, \ldots, v_{s}^{\delta_{v_{s}}-2}\right\}$, where in $\mathcal{N}^{m}$ each node $v$ has multiplicity $\delta_{v}-2$. Since $\sum_{v \in \mathcal{N}}\left(\delta_{v}-2\right)=|\mathcal{E}|-2$, $\mathcal{N}^{m}$ contains $|\mathcal{E}|-2$ symbols. Let $\mathcal{I}^{m} \subset \mathcal{N}^{m}$ be a non-trivial sub-multiset, $\mathcal{I}^{m}=$ $\left\{v_{i_{1}}^{k_{i_{1}}}, \ldots, v_{i_{r}}^{k_{i_{r}}}\right\}$, where $\left\{v_{i_{1}}, \ldots, v_{i_{r}}\right\}=\mathcal{I} \subset \mathcal{N}$ and $0<k_{i_{j}} \leq \delta_{v_{i_{j}}}-2$ for all $j$, and $0<r \leq s$. To such $\mathcal{I}^{m}$ we define $\left|\mathcal{I}^{m}\right|:=\sum_{j} k_{i_{j}},\left(\begin{array}{c}\delta-2 \\ k_{\mathcal{I}}\end{array}\right):=\prod_{j}\left(\begin{array}{c}\delta_{v_{i}}-2 \\ k_{i_{j}}\end{array}\right)$, the cycle $l^{\prime}\left(\mathcal{I}^{m}\right)=\sum_{j} k_{i_{j}} E_{v_{i j}}^{*}$ with $E^{*}$-support $\mathcal{I}$, and the polytope $\widetilde{\mathcal{P}}_{\mathcal{I}^{m}}:=\widetilde{\mathcal{P}}_{\mathcal{I}}^{\left(l^{\prime}\left(\mathcal{I}^{m}\right)\right)}$.

Note that $\widetilde{\mathcal{P}}_{\mathcal{N}^{m}}=\widetilde{\mathcal{P}}_{\mathcal{N}}^{\left(l^{\prime}\left(\mathcal{N}^{m}\right)\right)}=\widetilde{\mathcal{P}}_{\mathcal{N}}^{\left(l_{\text {top }}^{\prime}\right)}=\widetilde{\mathcal{P}}_{\mathcal{N}}^{\text {top }}$.

Recall that all these polytopes $\widetilde{\mathcal{P}}_{\mathcal{I}^{m}}$ sit in $\mathbb{R}_{\geq 0}^{|\mathcal{E}|}$, hence are $|\mathcal{E}|$-dimensional; their number is bounded from above by the cardinality of the index set $\mathcal{I}^{m}$, namely $-1+$ $\prod_{v}\left(\delta_{v}-1\right)$, however some of the polytopes might contain no lattice points.

Theorem 6.2.5 For any concave dilated polytope of type $\widetilde{\mathcal{P}}_{\mathcal{I}}^{\left(l^{\prime}\right)}$ we denote by $R_{h}\left(\widetilde{\mathcal{P}}_{\mathcal{I}}^{\left(l^{\prime}\right)}\right)$ the number of integral points in $\widetilde{\mathcal{P}}_{\mathcal{I}}^{\left(l^{\prime}\right)} \cap \rho^{-1}\left(\left[l^{\prime}\right]-h\right)$ with all coordinates strictly positive. Then

$$
-\mathfrak{s w}_{h}^{\text {norm }}(M)=\sum_{\emptyset \neq \mathcal{I}^{m} \subset \mathcal{N}^{m}}(-1)^{|\mathcal{E}|-\left|\mathcal{I}^{m}\right|} \cdot\left(\begin{array}{c}
\delta-2 \\
k_{\mathcal{I}^{m}}
\end{array}\right) \cdot R_{h}\left(\widetilde{\mathcal{P}}_{\mathcal{I}^{m}}\right) .
$$


Proof We need to compute $\mathcal{P}_{h, \mathcal{N}}^{+}(\mathbf{1})$. Let us fix some $\mathcal{I} \subset \mathcal{V}$, and we reformulate $\mathcal{P}_{h, \mathcal{I}}^{+}\left(\mathbf{t}_{\mathcal{I}}\right)$.

Sometimes is more convenient to calculate $\mathcal{P}_{h, \mathcal{I}}^{+}\left(\mathbf{t}_{\mathcal{I}}\right)$ via the truncation of the series $\mathcal{Z}_{\left[Z_{K}\right]-h}\left(\mathbf{t}_{\mathcal{I}}\right)$, the 'dual' polynomial part

$$
\check{P}_{h, \mathcal{I}}^{+}\left(\mathbf{t}_{\mathcal{I}}\right):=\sum_{\substack{\left[l^{\prime}\right]=\left[Z_{K}\right]-h \\ l^{\prime}\left|\mathcal{I} \nsucc\left(Z_{K}-E\right)\right|_{\mathcal{I}}}} z\left(l^{\prime}\right) \mathbf{t}_{\mathcal{I}}^{l^{\prime}}
$$

using the defining identity (5.3.1)

$$
P_{h, \mathcal{I}}^{+}\left(\mathbf{t}_{\mathcal{I}}\right)=\mathbf{t}_{\mathcal{I}}^{Z_{K}-E} \check{P}_{h, \mathcal{I}}^{+}\left(\mathbf{t}_{\mathcal{I}}^{-1}\right)
$$

Then (6.2.7) and (2.2.1) gives for the dual polynomial part

$$
\check{P}_{h, \mathcal{I}}^{+}\left(\mathbf{t}_{\mathcal{I}}\right)=\sum(-1)^{\sum_{v \in \mathcal{N}} k_{v}} \cdot\left(\begin{array}{c}
\delta-2 \\
k
\end{array}\right) \mathbf{t}_{\mathcal{I}}^{\sum_{v \in \mathcal{N}} k_{v} E_{v}^{*}+l(\mathbf{y})}
$$

where $\left(\begin{array}{c}\delta-2 \\ k\end{array}\right):=\prod_{v \in \mathcal{N}}\left(\begin{array}{c}\delta_{v}-2 \\ k_{v}\end{array}\right)$ and the sum runs over all $0 \leq k_{v} \leq \delta_{v}-2$ for every $v \in \mathcal{N}$ and $\mathbf{y} \in \mathbb{Z}_{\geq 0}^{|\mathcal{E}|} \cap \rho^{-1}\left(\left[Z_{K}-\sum_{v \in \mathcal{N}} k_{v} E_{v}^{*}\right]-h\right)$ such that $\left.\mathfrak{l}(\mathbf{y})\right|_{\mathcal{I}} \nsucc\left(Z_{K}-E-\right.$ $\left.\sum_{v \in \mathcal{N}} k_{v} E_{v}^{*}\right)\left.\right|_{\mathcal{I}}$

Now apply (6.2.8), (2.1.1) and replace $k_{v}$ by $\delta_{v}-2-k_{v}$ in order to get for the polynomial part

$$
P_{h, \mathcal{I}}^{+}\left(\mathbf{t}_{\mathcal{I}}\right)=(-1)^{|\mathcal{E}|} \sum(-1)^{\sum_{v \in \mathcal{N}} k_{v}}\left(\begin{array}{c}
\delta-2 \\
k
\end{array}\right) \cdot \mathbf{t}_{\mathcal{I}}^{\sum_{\mathcal{N}} k_{v} E_{v}^{*}-\mathfrak{l}(\mathbf{y})},
$$

where the sum runs over all $0 \leq k_{v} \leq \delta_{v}-2$ and $\mathbf{y} \in \widetilde{\mathcal{P}}_{\mathcal{V}}^{\left(\sum_{v \in \mathcal{N}} k_{v} E_{v}^{*}\right)} \cap$ $\rho^{-1}\left(\left[\sum_{v \in \mathcal{N}} k_{v} E_{v}^{*}\right]-h\right) \cap \mathbb{Z}_{>0}^{|\mathcal{E}|}$.

If we specify $\mathcal{I}=\mathcal{N}$, Lemma 6.2.2 implies that the sum in (6.2.10) runs over all $\mathcal{I}^{m} \subset \mathcal{N}^{m}, \mathcal{I}^{m} \neq \emptyset$, and lattice points of $\widetilde{P}_{\mathcal{I}^{m}} \cap \rho^{-1}\left(\left[\sum_{I} k_{v} E_{v}^{*}\right]-h\right)$ with all coordinates being strictly positive. Now, by Corollary 5.3.3 the formula (6.2.6) follows.

Remark 6.2.11 The above statement is rather similar with the geometric genus formula of Khovanskii [18] and Morales [29] for Newton non-degenerate isolated complete intersection singularities (supporting the intimate relationship between these two invariants), aside from the fact that the polytopes in the analytic theory are built via Minkowski sum from the 'elementary' ones.

Corollary 6.2.12 If the conditions $\left.Z_{K}\right|_{\mathcal{N}} \leq\left. E_{v}^{*}\right|_{\mathcal{N}}$ for every node $v \in \mathcal{N}$ are satisfied then

$$
-\mathfrak{s w}_{h}^{\text {norm }}(M)=R_{h}\left(\widetilde{\mathcal{P}}_{\mathcal{N}^{m}}\right)=R_{h}\left(\widetilde{\mathcal{P}}_{\mathcal{N}}^{\text {top }}\right)
$$


Moreover, the polynomial part is

$$
P_{h, \mathcal{N}}^{+}\left(\mathbf{t}_{\mathcal{N}}\right)=\sum \mathbf{t}_{\mathcal{N}}^{Z_{K}-E-l(\mathbf{y})}
$$

where the sum is over $\mathbf{y} \in \rho^{-1}\left(\left[Z_{K}\right]-h\right)$ and $\mathbf{y}+(1, \ldots, 1) \in \widetilde{\mathcal{P}}_{\mathcal{N}}^{\text {top }} \backslash \widetilde{\mathcal{G}}_{\mathcal{N}}^{\text {co }}$.

Proof In (6.2.6) only the submultiset $\mathcal{I}^{m}=\mathcal{N}^{m}$ has non-trivial contribution. Indeed, in the 'symmetric expression' (6.2.9) each $k_{v}$ should be zero, since $\left.\mathfrak{l}(\mathbf{y})\right|_{\mathcal{I}} \nsucc\left(Z_{K}-\right.$ $\left.E-\sum_{v \in \mathcal{N}} k_{v} E_{v}^{*}\right)\left.\right|_{\mathcal{I}}$.

Example 6.2.13 Let $M$ be a negative definite Seifert fibered rational homology sphere, ie. $\Gamma$ is a star-shaped tree. Assume that the central node $v_{0}$ has $d \geq 3$ legs, each with determinant $\alpha_{i} \geq 2(i=1, \ldots, d)$, and let $e$ be the orbifold Euler number of $M$. Then by the formula (2.1.1) one calculates $\left.\left(Z_{K}-E\right)\right|_{v_{0}}=\left(d-2-\sum_{i} 1 / \alpha_{i}\right) /|e|$; and one also has $\left.E_{v_{0}}^{*}\right|_{v_{0}}=1 /|e|$ (cf. [20, 6.1.3], [31, 11.1]). Hence the assumption of Corollary 6.2 .12 reads as

$$
d-3<\sum_{i=1}^{d} \frac{1}{\alpha_{i}} .
$$

Notice that this cannot happen if $d \geq 6$ and it is always satisfied for $d=3$. If (6.2.14) is satisfied then one needs exactly one polytope in Theorem 6.2.5, otherwise more.

Assume next that $\left\{\alpha_{i}\right\}_{i}$ are pairwise relative prime, or, equivalently, that $M$ is an integral homology sphere (link of a Brieskorn complete intersection). Then for $d=3$ by Corollary 6.2 .12 we recover the well-known result that the normalized Casson invariant of $M\left(\alpha_{1}, \alpha_{2}, \alpha_{3}\right)$ is the number of lattice points in the polytope $\mathcal{P}_{v_{0}}=\left\{\mathbf{y} \in \mathbb{R}_{>0}^{3}: \sum_{i=1}^{3} y_{i} / \alpha_{i} \leq 1\right\}$ but not on the coordinate faces. In fact, in this case $\mathcal{P}_{v_{0}}$ coincides with the Newton polytope associated with the equation of the Brieskorn germ $\left(\left\{\sum_{i=1}^{3} y_{i}^{\alpha_{i}}=0\right\}, 0\right)$. For arbitrary $d \geq 3$ one has

$$
\begin{aligned}
& -\mathfrak{s w}_{0}^{\text {norm }}(M) \\
& =\sum_{0<k \leq d-2}(-1)^{d-2-k}\left(\begin{array}{c}
d-2 \\
k
\end{array}\right) \cdot \text { cardinality of }\left\{\left(y_{i}\right)_{i} \in\left(\mathbb{Z}_{>0}\right)^{d} \mid \sum_{i} y_{i} / \alpha_{i} \leq k\right\} .
\end{aligned}
$$

In this case the common Newton polytope of the $(d-2)$-Brieskorn equations is $\mathcal{P}_{v_{0}}=\left\{\mathbf{y} \in \mathbb{R}_{\geq 0}^{d}: \sum_{i=1}^{d} y_{i} / \alpha_{i} \leq 1\right\}$, hence the result fits perfectly with the geometric genus formula of [29].

For more details and different other aspects see [10,15,20,36,40,45].

Example 6.2.15 Let $M$ be the manifold associated with the following plumbing graph $\Gamma:$

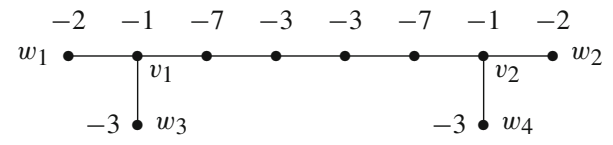

$M$ is realized e.g. as the link of the Newton non-degenerate hypersurface singularity $f:\left(\mathbb{C}^{3}, 0\right) \rightarrow(\mathbb{C}, 0), f(x, y, z)=x^{13}+y^{13}+z^{3}+x^{2} y^{2}$. In the sequel, following 
the above discussions, we present the computation of the (dual) polynomial part using the lattice points of the topological polytope.

From the negative inverse of the intersection matrix we read the linear forms

$$
\begin{aligned}
& \mathfrak{l}_{v_{j}}: \mathbb{R}^{4}=\mathbb{R}\left\langle E_{w_{1}}, \ldots, E_{w_{4}}\right\rangle \rightarrow \mathbb{R}: \mathfrak{l}_{v_{1}}(\mathbf{y})=\langle(33,6,22,4), \mathbf{y}\rangle \text { and } \mathfrak{l}_{v_{2}}(\mathbf{y}) \\
& \quad=\langle(6,33,4,22), \mathbf{y}\rangle
\end{aligned}
$$

associated with the nodes $v_{1}$ and $v_{2}$. Moreover, one gets $\left.l_{\text {top }}^{\prime}\right|_{\mathcal{N}}=(78,78)$ and $\left.Z_{K}\right|_{\mathcal{N}}=(14,14)$ in the $\left(E_{v_{1}}, E_{v_{2}}\right)$-basis, hence the topological polytope is given by

$$
\widetilde{\mathcal{P}}_{\mathcal{N}}^{\text {top }}:=\left\{\mathbf{y} \in\left(\mathbb{R}_{\geq 0}\right)^{4}:\langle(33,6,22,4), \mathbf{y}\rangle \leq 78 \text { or }\langle(6,33,4,22), \mathbf{y}\rangle \leq 78\right\}
$$

The group $H \simeq \mathbb{Z}_{3}$ is generated by eg. $h_{1}=\left[E_{w_{3}}^{*}\right]$ and one can see by calculations that $\left[Z_{K}\right]=0$, or by the fact that $M$ is the link of a Gorenstein (in this case hypersurface) singularity. Moreover, $\left[E_{v_{1}}^{*}\right]=\left[E_{v_{2}}^{*}\right]=0$ too.

First we compute the 'dual polynomials' $\check{P}_{h, \mathcal{N}}^{+}(\mathbf{t}) .(6.2 .9)$ implies that

$$
\check{P}_{h, \mathcal{N}}^{+}(\mathbf{t})=\sum_{\star} \mathbf{t}^{\mathfrak{l}(\mathbf{y})}-\sum_{\star_{1}} \mathbf{t}^{E_{v_{1}}^{*}+\mathfrak{l}(\mathbf{y})}-\sum_{\star_{2}} \mathbf{t}^{E_{v_{2}}^{*}+\mathfrak{l}(\mathbf{y})},
$$

where $\mathbf{t}:=\mathbf{t}_{\mathcal{N}}=\left(t_{v_{1}}, t_{v_{2}}\right)$ and the sums run under the following conditions:

$$
\begin{aligned}
& (\star): \quad \mathbf{y} \in \mathbb{Z}^{4} \cap \rho^{-1}(-h) \text { and } \mathfrak{l}_{\mathcal{N}}(\mathbf{y}) \nsucc(13,13) ; \\
& \left(\star_{1}\right): \mathbf{y} \in \mathbb{Z}^{4} \cap \rho^{-1}(-h) \text { and } \mathfrak{l}_{\mathcal{N}}(\mathbf{y}) \nsucc(-53,1) ; \\
& \left(\star_{2}\right): \mathbf{y} \in \mathbb{Z}^{4} \cap \rho^{-1}(-h) \text { and } \mathfrak{l}_{\mathcal{N}}(\mathbf{y}) \nsucc(1,-53) .
\end{aligned}
$$

Then it can be checked that for $h=0$ the lattice points satisfying the condition $(\star)$ are $(0,0,0,0),(1,0,0,0),(0,1,0,0),(2,0,0,0),(0,2,0,0),(0,0,3,0)$ and $(0,0,0,3)$, creating the monomials $1, \mathbf{t}^{(33,6)}, \mathbf{t}^{(6,33)}, \mathbf{t}^{(66,12)}, \mathbf{t}^{(12,66)}, \mathbf{t}^{(66,12)}$ and $\mathbf{t}^{(12,66)}$ respectively. For $\left(\star_{1}\right)$ and $\left(\star_{2}\right)$ the only lattice point is $(0,0,0,0)$ with the associated monomials $\mathbf{t}^{(66,12)}$ and $\mathbf{t}^{(12,66)}$. Therefore, $\check{P}_{0, \mathcal{N}}^{+}(\mathbf{t})=1+\mathbf{t}^{(33,6)}+\mathbf{t}^{(6,33)}+$ $\mathbf{t}^{(66,12)}+\mathbf{t}^{(12,66)}$ which by (6.2.8) implies

$$
\begin{aligned}
P_{0, \mathcal{N}}^{+}(\mathbf{t})= & \mathbf{t}^{(13,13)} \cdot \check{P}_{h, \mathcal{N}}^{+}\left(\mathbf{t}^{-1}\right)=\mathbf{t}^{(1,-53)}+\mathbf{t}^{(-53,1)}+\mathbf{t}^{(7,-20)}+\mathbf{t}^{(-20,7)} \\
& +\mathbf{t}^{(13,13)} \text { and }-\mathfrak{s w}_{0}^{\text {norm }}(M)=5 .
\end{aligned}
$$

Similarly, for $h_{1}$ we get the lattice points (from $\left.\rho^{-1}\left(2 h_{1}\right)\right)(0,0,0,1),(0,0,2,0)$ and $(0,1,0,1)$ satisfying $(\star)$ and associating the monomials $\mathbf{t}^{(4,22)}, \mathbf{t}^{(44,8)}$ and $\mathbf{t}^{(10,55)}$. Hence, $\check{P}_{h_{1}, \mathcal{N}}^{+}(\mathbf{t})=\mathbf{t}^{(4,22)}+\mathbf{t}^{(44,8)}+\mathbf{t}^{(10,55)}$ and $-\mathfrak{s w}_{h_{1}}^{\text {norm }}(M)=3$. Symmetrically, one has $\check{P}_{2 h_{1}, \mathcal{N}}^{+}(\mathbf{t})=\mathbf{t}^{(22,4)}+\mathbf{t}^{(8,44)}+\mathbf{t}^{(55,10)}$ and $-\mathfrak{s w}_{2 h_{1}}^{\text {norm }}(M)=3$. 


\subsection{Minkowski sums of polyhedrons associated with nodes}

For all the polytopes $\mathcal{P}$ considered in this subsection we denote by $\mathcal{P}^{\triangleleft}:=\mathcal{P} \backslash\left(\mathcal{F} \backslash \mathcal{G}^{c o}\right)$ the corresponding semi-open polytope and define the unbounded convex polyhedron $\mathcal{P}^{+}:=\mathbb{R}_{\geq 0}^{|\mathcal{E}|} \backslash \mathcal{P}^{\triangleleft}$. In the sequel we discuss relations between polyhedrons of type $\widetilde{\mathcal{P}}_{\mathcal{I}^{m}}^{+}$and Minkowski sums of $\left(\mathcal{P}_{v}^{\left(E_{v}^{*}\right)}\right)^{+}$. In particular, we prove for special cases a Khovanskii-Morales type IELP expression for the normalized Seiberg-Witten invariants using Minkowski sums.

Lemma 6.3.1 For any $v \in \mathcal{N}$ let $\mathcal{E}_{v}$ be the set of those end-vertices $w \in \mathcal{E}$ for which the unique minimal connected full subgraph $[w, v]$ does not contain any other nodes than $v$. Then for any $v^{\prime} \in \mathcal{N}$ we have

$$
\frac{\left.E_{w}^{*}\right|_{v}}{\left.E_{w}^{*}\right|_{v^{\prime}}}=\frac{\left.E_{v}^{*}\right|_{v}}{\left.E_{v^{\prime}}^{*}\right|_{v}}=: \lambda_{v v^{\prime}} \quad \text { for any } w \in \mathcal{E}_{v} .
$$

Moreover, if $v^{\prime \prime} \in \mathcal{N}$ is a vertex of the subgraph $\left[v, v^{\prime}\right]$ for some $v, v^{\prime} \in \mathcal{N}$ then $\lambda_{v v^{\prime}}=\lambda_{v v^{\prime \prime}} \cdot \lambda_{v^{\prime \prime} v^{\prime}}$.

Proof It follows from the formula of [14] expressing $-\left(E_{v^{\prime}}^{*}, E_{v^{\prime \prime}}^{*}\right)$ as the fraction of the determinant (of the negative intersection form) of the graph $\Gamma \backslash\left[v^{\prime}, v^{\prime \prime}\right]$ and $|H|$. Here, $\left[v^{\prime}, v^{\prime \prime}\right]$ denotes the unique minimal connected subgraph containing the vertices $v^{\prime}$ and $v^{\prime \prime}$.

Proposition 6.3.3 (a) For any $k_{v} \in \mathbb{Z}_{\geq 0}, v \in \mathcal{N}$ write $l^{\prime}:=\sum_{v} k_{v} E_{v}^{*}$. Then

$$
\sum_{v \in \mathcal{N}} k_{v}\left(\mathcal{P}_{v}^{\left(E_{v}^{*}\right)}\right)^{+} \subset\left(\widetilde{\mathcal{P}}_{s p^{*}\left(l^{\prime}\right)}^{\left(l^{\prime}\right)}\right)^{+}
$$

(b) Let $v_{1}, v_{2} \in \mathcal{N}$ be two neighbouring nodes in $\Gamma$, ie. the minimal connected subgraph $\left[v_{1}, v_{2}\right]$ does not contain any other nodes. Then $k_{v_{1}}\left(\mathcal{P}_{v_{1}}^{\left(E_{v_{1}}^{*}\right)}\right)^{+}+$ $k_{v_{2}}\left(\mathcal{P}_{v_{2}}^{\left(E_{v_{2}}^{*}\right)}\right)^{+}=\left(\widetilde{\mathcal{P}}_{\left\{v_{1}, v_{2}\right\}}^{\left(k_{v_{1}} E_{v_{1}}^{*}+k_{v_{2}} E_{v_{2}}^{*}\right)}\right)^{+}$.

Proof (a) By definition of $\left(\mathcal{P}_{v}^{\left(E_{v}^{*}\right)}\right)^{+}$follows that the Minkowski sum $\sum_{v \in \mathcal{N}} k_{v}$ $\left(\mathcal{P}_{v}^{\left(E_{v}^{*}\right)}\right)^{+}$is also a convex polyhedron bounded from below by the convex hull of its vertices and the coordinate hyperplanes. Any vertex $\mathbf{v}_{\mathbf{k}}$ of the Minkowski sum can be written as $\sum_{v \in \mathcal{N}} \sum_{j=1}^{k_{v}} \mathbf{v}_{v}^{j}$, where $\mathbf{v}_{v}^{j}$ is a certain vertex of the polyhe$\operatorname{dron}\left(\mathcal{P}_{v}^{\left(E_{v}^{*}\right)}\right)^{+}=\left\{\mathbb{R}_{\geq 0}^{|\mathcal{E}|}:\left.\sum_{e} y_{e} E_{e}^{*}\right|_{v} \geq\left. E_{v}^{*}\right|_{v}\right\}$. Hence $\mathbf{v}_{v}^{j}$ is a vector whose entries are all zero except exactly one, say with index $e$, and that entry is $\left(\left.E_{v}^{*}\right|_{v}\right) /\left(\left.E_{e}^{*}\right|_{v}\right)$. Hence $\mathfrak{l}\left(\mathbf{v}_{v}^{j}\right)=\left(\left.E_{v}^{*}\right|_{v}\right) /\left(\left.E_{e}^{*}\right|_{v}\right) E_{e}^{*}$ for some $e \in \mathcal{E}$.

Now, similarly as in the proof of Lemma 6.2.2 we consider the set $\{v\}$ (this plays the role of $\mathcal{I}$ in that proof), and the subgraph $\Gamma_{1}:=\Gamma \backslash v$, and we notice that $e$ is contained in $\Gamma_{1}$ (since $v$ is a node and $e$ an end-vertex). Hence, as in that lemma, one has an identity of cycles $(\dagger) E_{e}^{*}-r E_{v}^{*}=i\left(E_{e}^{*}\left(\Gamma_{1}\right)\right)$. In particular, $E_{e}^{*}-r E_{v}^{*} \geq 0$. 
On the other hand, intersected $(\dagger)$ with $E_{v}^{*}$ we get that $\left.E_{e}^{*}\right|_{v}-\left.r E_{v}^{*}\right|_{v}=0$, or $\left(\left.E_{v}^{*}\right|_{v}\right) /\left(\left.E_{e}^{*}\right|_{v}\right)=r^{-1}$. Hence $\mathfrak{l}\left(\mathbf{v}_{v}^{j}\right)=r^{-1} E_{e}^{*} \geq E_{v}^{*}$.

This implies the inequality $\mathfrak{l}_{\mathcal{N}}\left(\mathbf{v}_{\mathbf{k}}\right) \geq\left.\sum_{v \in \mathcal{N}} k_{v} E_{v}^{*}\right|_{\mathcal{N}}$ for all vertices of the Minkowski sum, hence for all $\sum_{v \in \mathcal{N}} k_{v}\left(\mathcal{P}_{v}^{\left(E_{v}^{*}\right)}\right)^{+}$too.

(b) Since the coefficients $k_{v_{1}}, k_{v_{2}}$ do not change the combinatorial type of neither of the two polyhedrons (see eg. [17] for Minkowski sums) we assume that $k_{v_{1}}=k_{v_{2}}=1$. By (a) we have to prove that the compact faces of $\left(\widetilde{\mathcal{P}}_{\left\{v_{1}, v_{2}\right\}}^{\left(E_{v_{2}}^{*}+E_{v_{2}}^{*}\right)}\right)^{+}$can be written as Minkowski sums of compact faces of $\left(\mathcal{P}_{v_{1}}^{\left(E_{v_{1}}^{*}\right)}\right)^{+}$and $\left(\mathcal{P}_{v_{2}}^{\left(E_{v_{2}}^{*}\right)}\right)^{+}$. By the definition of $\widetilde{\mathcal{P}}_{\left\{v_{1}, v_{2}\right\}}^{\left(E_{v_{2}}^{*}+E_{2}^{*}\right)}$ these compact faces are given by the hyperplanes $\mathfrak{l}_{v_{1}}=\left.\left(E_{v_{1}}^{*}+E_{v_{2}}^{*}\right)\right|_{v_{1}}$, $\mathfrak{l}_{v_{2}}=\left.\left(E_{v_{1}}^{*}+E_{v_{2}}^{*}\right)\right|_{v_{2}}$ and their intersections. Now, consider the stratification $\mathcal{E}=$ $\mathcal{E}_{1} \amalg \mathcal{E}_{2}$ of the set of ends so that $\mathcal{E}_{i}$ are those end-vertices which are contained in the same connected subgraph as $v_{i}$ if we cut out $\left[v_{1}, v_{2}\right] \backslash\left\{v_{1}, v_{2}\right\}$ from $\Gamma$. We denote by $\mathbf{v}_{i}^{(j)}$ the (non-zero) vertex of $\mathcal{P}_{v_{i}}^{\left(E_{v_{i}}^{*}\right)}$ which are situated on the coordinate axis corresponding to $w \in \mathcal{E}_{j}$ and let $F\left\langle\mathbf{v}_{i, w}^{(j)}\right\rangle$ be the face generated by the vertices of type $\mathbf{v}_{i, w}^{(j)}$ for some fixed $i$ and $j$.

Then our claim is that the compact facets of $\widetilde{\mathcal{P}}_{\left\{v_{1}, v_{2}\right\}}^{\left(E_{v_{2}}^{*}+E_{2}^{*}\right)}$ are given by the Minkowski sums $F\left\langle\mathbf{v}_{1}^{(1)}, \mathbf{v}_{1}^{(2)}\right\rangle+F\left\langle\mathbf{v}_{2}^{(2)}\right\rangle, F\left\langle\mathbf{v}_{1}^{(1)}\right\rangle+F\left\langle\mathbf{v}_{1}^{(2)}, \mathbf{v}_{2}^{(2)}\right\rangle$, and their intersection is given by $F\left\langle\mathbf{v}_{1}^{(1)}\right\rangle+F\left\langle\mathbf{v}_{2}^{(2)}\right\rangle$. Indeed, using Lemma 6.3 .1 we deduce that

$$
\mathfrak{l}_{v_{1}}=\mathfrak{l}_{v_{1}}\left|\mathcal{E}_{1}+\left(1 / \lambda_{v_{2} v_{1}}\right) \mathfrak{l}_{v_{2}}\right| \mathcal{E}_{2} \quad \text { and } \quad \mathfrak{l}_{v_{2}}=\left(1 / \lambda_{v_{1} v_{2}}\right) \mathfrak{l}_{v_{1}}\left|\mathcal{E}_{1}+\mathfrak{l}_{v_{2}}\right| \mathcal{E}_{2}
$$

where $\left.\mathfrak{l}_{v_{i}}\right|_{\mathcal{E}_{i}}(\mathbf{y})=\left.\sum_{w \in \mathcal{E}_{i}} y_{w} E_{w}^{*}\right|_{v_{i}}$. Hence, $\mathfrak{l}_{v_{1}}\left(F\left\langle\mathbf{v}_{1}^{(1)}, \mathbf{v}_{1}^{(2)}\right\rangle+F\left\langle\mathbf{v}_{2}^{(2)}\right\rangle\right)=\left.E_{v_{1}}^{*}\right|_{v_{1}}+$ $\left.\left(1 / \lambda_{21}\right) E_{v_{2}}^{*}\right|_{v_{2}}=\left.\left(E_{v_{1}}^{*}+E_{v_{2}}^{*}\right)\right|_{v_{1}}$. Similarly, one has $\mathfrak{l}_{v_{2}}\left(F\left\langle\mathbf{v}_{1}^{(1)}\right\rangle+F\left\langle, \mathbf{v}_{1}^{(2)} \mathbf{v}_{2}^{(2)}\right\rangle\right)=$ $\left.\left(E_{v_{1}}^{*}+E_{v_{2}}^{*}\right)\right|_{v_{2}}$ and $F\left\langle\mathbf{v}_{1}^{(1)}\right\rangle+F\left\langle\mathbf{v}_{2}^{(2)}\right\rangle$ satisfies both equations.

Corollary 6.3.4 For a graph $\Gamma$ with two nodes the formula (6.2.6) can be interpreted by Minkowski sums in the following way

$$
-\mathfrak{s w}_{h}^{\text {norm }}(M)=\sum_{\emptyset \neq \mathcal{I}^{m} \subset \mathcal{N}^{m}}(-1)^{|\mathcal{E}|-\left|\mathcal{I}^{m}\right|} \cdot\left(\begin{array}{c}
\delta-2 \\
k_{\mathcal{I}^{m}}
\end{array}\right) \cdot \bar{R}_{h}\left(\sum_{j} k_{i_{j}}\left(\mathcal{P}_{v_{i_{j}}}^{\left(E_{i_{j}}^{*}{ }^{*}\right.}\right)^{+}\right)
$$

where $\bar{R}_{h}(\mathcal{P})$ is a $\rho^{-1}\left(\left[l^{\prime}\right]-h\right)$-lattice point counting with all coordinates being strictly positive and not in the interior of $\mathcal{P}$.

The next example has two messages. First, it is an example with three nodes when the corresponding identity from Proposition 6.3.3(b) is not valid. On the other hand, for this example the number of lattice points in the two (distinct) polytopes agree. This fact suggests that though the validity of Proposition 6.3.3(b) cannot be expected in general, a Khovanskii-Morales type IELP formula for the normalized Seiberg-Witten invariants using Minkowski sums may exist. 
Example 6.3.5 Consider the following graph with trivial $H$ :

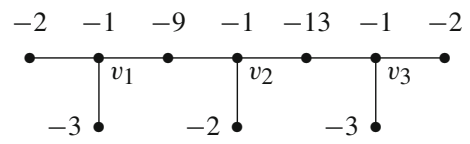

The normalized Seiberg-Witten invariant has been calculated in [22, 6.5] using the polynomial $P_{1}^{+}$(see 5.1 for details) developed in the very same article. Now we restrict our attention to the Minkowski sums of polyhedrons $\left(\mathcal{P}_{v_{i}}^{\left(E_{v_{i}}^{*}\right)}\right)^{+}$.

The polytopes associated with the nodes are $\mathcal{P}_{v_{1}}^{\left(E_{v_{1}}^{*}\right)}=\left\{\mathbf{y} \in \mathbb{R}^{5}:\langle(93,62,42,36,24)\right.$, $\mathbf{y}\rangle \leq 186\}, \mathcal{P}_{v_{2}}^{\left(E_{v_{2}}^{*}\right)}=\left\{\mathbf{y} \in \mathbb{R}^{5}:\langle(42,28,21,18,12), \mathbf{y}\rangle \leq 42\right\}$ and $\mathcal{P}_{v_{3}}^{\left(E_{v_{3}}^{*}\right)}=$ $\left\{\mathbf{y} \in \mathbb{R}^{5}:\langle(36,24,18,21,14), \mathbf{y}\rangle \leq 42\right\}$. The Minkowski sums can be described as follows: $\left(\mathcal{P}_{v_{1}}^{\left(E_{v_{1}}^{*}\right)}\right)^{+}+\left(\mathcal{P}_{v_{2}}^{\left(E_{v_{2}}^{*}\right)}\right)^{+}=\left(\widetilde{\mathcal{P}}_{\left\{v_{1}, v_{2}\right\}}^{\left(E_{v_{1}}^{*}+E_{v_{2}}^{*}\right)}\right)^{+},\left(\mathcal{P}_{v_{2}}^{\left(E_{v_{2}}^{*}\right)}\right)^{+}+\left(\mathcal{P}_{v_{3}}^{\left(E_{v_{3}}^{*}\right)}\right)^{+}=$ $\left(\widetilde{\mathcal{P}}_{\left\{v_{2}, v_{3}\right\}}^{\left(E_{v_{3}}^{*}+E_{v_{3}}^{*}\right)}\right)^{+}$follows from Proposition 6.3.3(b), while

$$
\left(\mathcal{P}_{v_{1}}^{\left(E_{v_{1}}^{*}\right)}\right)^{+}+\left(\mathcal{P}_{v_{3}}^{\left(E_{v_{3}}^{*}\right)}\right)^{+}=\left(\widetilde{\mathcal{P}}_{\left\{v_{1}, v_{3}\right\}}^{\left(E_{v_{1}}^{*}+E_{v_{3}}^{*}\right)} \cup \mathcal{P}_{v_{1} v_{3}}^{\left(l^{\prime}\right)}\right)^{+}
$$

where the extra polytope is given by

$$
\begin{aligned}
\mathcal{P}_{v_{1} v_{3}}^{\left(l^{\prime}\right)} & :=\left\{\mathbf{y} \in \mathbb{R}^{5}:\langle(93,62,42,49,98 / 3), \mathbf{y}\rangle \leq\left. l^{\prime}\right|_{v_{1}}\right. \\
& \left.:=\left.\left(E_{v_{1}}^{*}+\left(\lambda_{v_{2} v_{3}} \lambda_{v_{3} v_{1}} / \lambda_{v_{2} v_{1}}\right) \cdot E_{v_{3}}^{*}\right)\right|_{v_{1}}=284\right\}
\end{aligned}
$$

and $\lambda_{v_{i} v_{j}}$ are the constants from Lemma 6.3.1. Similarly, one has

$$
\left(\mathcal{P}_{v_{1}}^{\left(E_{v_{1}}^{*}\right)}\right)^{+}+\left(\mathcal{P}_{v_{2}}^{\left(E_{v_{2}}^{*}\right)}\right)^{+}+\left(\mathcal{P}_{v_{3}}^{\left(E_{v_{3}}^{*}\right)}\right)^{+}=\left(\widetilde{\mathcal{P}}_{\left\{v_{1}, v_{2}, v_{3}\right\}}^{\left(E_{v_{1}}^{*}+E_{v_{2}}^{*}+E_{v_{3}}^{*}\right)} \cup \mathcal{P}_{v_{1} v_{3}}^{\left(l^{\prime}+E_{v_{2}}^{*}\right)}\right)^{+}
$$

One can calculate $R_{0}\left(\widetilde{\mathcal{P}}_{\left\{v_{i}\right\}}\right)=R_{0}\left(\widetilde{\mathcal{P}}_{\left\{v_{2}, v_{3}\right\}}\right)=0, R_{0}\left(\widetilde{\mathcal{P}}_{\left\{v_{1}, v_{2}\right\}}\right)=1$, and we also find the identities $\bar{R}_{0}\left(\left(\mathcal{P}_{v_{1}}^{\left(E_{v_{1}}^{*}\right)}\right)^{+}+\left(\mathcal{P}_{v_{3}}^{\left(E_{v_{3}}^{*}\right)}\right)^{+}\right)=R_{0}\left(\widetilde{\mathcal{P}}_{\left\{v_{1}, v_{3}\right\}}\right)=1$ and $\bar{R}_{0}\left(\left(\sum_{i=1}^{3} \mathcal{P}_{v_{i}}^{\left(E_{v_{i}}^{*}\right)}\right)^{+}\right)=R_{0}\left(\widetilde{\mathcal{P}}_{\left\{v_{1}, v_{2}, v_{3}\right\}}\right)=15$. Hence,

$$
-\mathfrak{s w}_{h}^{\text {norm }}(M):=\sum_{\emptyset \neq I \subset\left\{v_{1}, v_{2}, v_{3}\right\}}(-1)^{5-|I|} \cdot \bar{R}_{0}\left(\sum_{i \in I}\left(\mathcal{P}_{v_{i}}^{\left(E_{v_{i}}^{*}\right)}\right)^{+}\right)=13 .
$$

Acknowledgements Open access funding provided by MTA Alfréd Rényi Institute of Mathematics (MTA RAMKI). TL was supported by ERCEA Consolidator Grant 615655-NMST and by the Basque Government through the BERC 2014-2017 program and by Spanish Ministry of Economy and Competitiveness MINECO: BCAM Severo Ochoa excellence accreditation SEV-2013-0323. AN was partially supported by ERC Adv. Grant LDTBud of A. Stipsicz at Rényi Institute of Math., Budapest. TL and AN were also supported by NKFIH Grant "Élvonal” (Frontier) KKP 126683. JN was partially supported by NKFIH Grant K119670, JN and AN were partially supported by NKFIH Grant K112735.

Open Access This article is distributed under the terms of the Creative Commons Attribution 4.0 International License (http://creativecommons.org/licenses/by/4.0/), which permits unrestricted use, distribution, 
and reproduction in any medium, provided you give appropriate credit to the original author(s) and the source, provide a link to the Creative Commons license, and indicate if changes were made.

\section{References}

1. A'Campo, N.: La fonction zêta d'une monodromie. Comment. Math. Helv. 50, 233-248 (1975)

2. Arnold, V.I., Gusein-Zade, S.M., Varchenko, A.N.: Singularities of differentiable maps, vol. 2. Monodromy and asymptotics of integrals. Modern Birkhäuser Classics. Birkhäuser/Springer, New York (2012). Translated from the Russian by Hugh Porteous (2012)

3. Beck, M.: Multidimensional Ehrhart reciprocity. J. Comb. Theory Ser. A 97, 187-194 (2002)

4. Bivià-Ausina, C.: Mixed Newton numbers and isolated complete intersection singularities. Proc. Lond. Math. Soc. 94(3), 749-771 (2007)

5. Braun, G., Némethi, A.: Surgery formula for Seiberg-Witten invariants of negative definite plumbed 3-manifolds. J. Reine Angew. Math. 638, 189-208 (2010)

6. Braun, G., Némethi, A.: Invariants of Newton non-degenerate surface singularities. Compos. Math. 143, 1003-1036 (2007)

7. Campillo, A., Delgado, F., Gusein-Zade, S.M.: The Alexander polynomial of a plane curve singularity via the ring of functions on it. Duke Math J. 117(1), 125-156 (2003)

8. Campillo, A., Delgado, F., Gusein-Zade, S.M.: Poincaré series of a rational surface singularity. Invent. Math. 155(1), 41-53 (2004)

9. Campillo, A., Delgado, F., Gusein-Zade, S.M.: Universal abelian covers of rational surface singularities and multi-index filtrations. Funk. Anal. Prilozhen. 42(2), 3-10 (2008)

10. Can, M.B., Karakurt, Ç.: Calculating Heegaard-Floer homology by counting lattice points in tetrahedra. Acta Math. Hungar. 144(1), 43-75 (2014)

11. Durfee, A.H.: The signature of smoothings of complex surface singularities. Math. Ann. 232(1), 85-98 (1978)

12. Ehrhart, E.: Sur un probléme de géométrie diophantienne linéaire I. \& II. J. Reine Angew. Math. 226, 25-49 (1967). (and 227 (1967), 25-49)

13. Ehrhart, E.: Démonstration de la loi de réciprocité du polyédre rationnel. J. C. R. Acad. Sci. Paris 265A, 91-94 (1967)

14. Eisenbud, D., Neumann, W.: Three-Dimensional Link Theory and Invariants of Plane Curve Singularities. Princeton University Press, Princeton (1985)

15. Fintushel, R., Stern, R.J.: Instanton homology of Seifert fibred homology three spheres. Proc. Lond. Math. Soc. (3) 61(1), 109-137 (1990)

16. Gompf, R. E., Stipsicz, A.: An introduction to 4-manifolds and Kirby calculus. Graduate Studies in Mathematics, vol. 20, Am. Math. Soc. (1999)

17. Gritzmann, P., Sturmfels, B.: Minkowski addition of polytopes: computational complexity and applications to Gröbner bases. SIAM J. Disc. Math. 6(2), 246-269 (1993)

18. Khovanskii, A.G.: Newton polyhedra, and the genus of complete intersections (Russian). Funk. Anal. Prilozhen. 12(1), 51-61 (1978)

19. Kouchnirenko, A.G.: Polyédres de Newton et nombres de Milnor. Invent. Math. 32(1), 1-31 (1976)

20. László, T., Némethi, A.: Ehrhart theory of polytopes and Seiberg-Witten invariants of plumbed 3manifolds. Geom. Topol. 18(2), 717-778 (2014)

21. László, T., Nagy, J., Némethi, A.: Surgery formulae for the Seiberg-Witten invariant of plumbed 3-manifold. Rev. Mat. Complut. (2019). https://doi.org/10.1007/s13163-019-00297-z

22. László, T., Szilágyi, Z.: On Poincaré series associated with links of normal surface singularities. Trans. AMS (2019). https://doi.org/10.1090/tran/7802

23. László, T., Szilágyi, Z.: Némethi's division algorithm for zeta-functions of plumbed 3-manifolds. Bull. Lond. Math. Soc. 50, 1035-1055 (2018)

24. Lim, Y.: Seiberg-Witten invariants for 3-manifolds in the case $b_{1}=0$ or 1 . Pac. J. Math. 195(1), 179-204 (2000)

25. Lescop, C.: Global surgery formula for the Casson-Walker invariant. Ann. of Math. Studies, vol. 140. Princeton Univ. Press (1996)

26. Macdonald, I.G.: Polynomials associated with finite cell-complexes. J. Lond. Math. Soc. 4(2), 181-192 (1971) 
27. Merle, M., Teissier, B.: Conditions d'adjonction, d'après DuVal. In: Séminaire sur les Singularités des Surfaces, Lecture Notes in Math., vol. 777. Springer, Berlin, pp. 229-245 (1980)

28. McMullen, P.: Lattice invariant valuations on rational polytopes. Arch. Math. (Basel) 31, 509-516 $(1978 / 79)$

29. Morales, M.: Polyèdre de Newton et genre géométrique d'une singularité intersection complète. Bull. Soc. Math. France 112(3), 325-341 (1984)

30. Némethi, A.: Five lectures on normal surface singularities, lectures at the Summer School in Low dimensional topology Budapest, Hungary, 1998; Bolyai Society Math. Studies, vol. 8, pp. 269-351 (1999)

31. Némethi, A.: On the Ozsváth-Szabó invariant of negative definite plumbed 3-manifolds. Geom. Topol. 9, 991-1042 (2005)

32. Némethi, A.: Graded Roots and Singularities. Singularities in Geometry and Topology, pp. 394-463. World Sci. Publ., Hackensack (2007)

33. Némethi, A.: Poincaré series associated with surface singularities. In: Singularities I, pp. 271-297, Contemp. Math., vol. 474, Amer. Math. Soc., Providence, RI (2008)

34. Némethi, A.: The Seiberg-Witten invariants of negative definite plumbed 3-manifolds. J. Eur. Math. Soc. 13, 959-974 (2011)

35. Némethi, A.: The cohomology of line bundles of splice-quotient singularities. Adv. Math. 229(4), 2503-2524 (2012)

36. Némethi, A., Nicolaescu, L.I.: Seiberg-Witten invariants and surface singularities. Geom. Topol. 6, 269-328 (2002)

37. Némethi, A., Okuma, T.: On the Casson invariant conjecture of Neumann-Wahl. J. Algebraic Geom. 18, 135-149 (2009)

38. Neumann, W., Wahl, J.: Casson invariant of links of singularities. Comment. Math. Helv. 65, 58-78 (1990)

39. Neumann, W., Wahl, J.: Complete intersection singularities of splice type as universal abelian covers. Geom. Topol. 9, 699-755 (2005)

40. Nicolaescu, L.: Lattice points inside rational simplices and the Casson invariant of Brieskorn spheres. Geom. Dedic. 88, 37-53 (2001)

41. Nicolaescu, L.: Seiberg-Witten invariants of rational homology 3-spheres. Commun. Contemp. Math. 6(6), 833-866 (2004)

42. Oka, M.: Principal zeta-function of nondegenerate complete intersection singularity. J. Fac. Sci. Univ. Tokyo Sect. IA Math. 37(1), 11-32 (1990)

43. Oka, M.: Non-degenerate complete intersection singularity. Actualités mathématiques, Hermann Éditeurs (1997)

44. Okuma, T.: The geometric genus of splice-quotient singularities. Trans. Am. Math. Soc. 360(12), 6643-6659 (2008)

45. Saveliev, N.: Invariants of Homology 3-Spheres. Encyclopaedia of Mathematical Sciences, vol. 140. Springer, Berlin (2002)

46. Sigurðsson, B: The geometric genus and Seiberg-Witten invariant of Newton nondegenerate surface singularities, Ph.D. thesis, Central European University, Budapest (2015)

47. Stanley, R.P.: Combinatorial reciprocity theorems. Adv. Math. 14, 194-253 (1974)

48. Szenes, A., Vergne, M.: Residue formulae for vector partitions and Euler-Maclaurin sums. Adv. Appl. Math. 30, 295-342 (2003)

49. Varchenko, A.: Zeta-function of monodromy and Newton's diagram. Invent. Math. 37(3), 253-262 (1976)

Publisher's Note Springer Nature remains neutral with regard to jurisdictional claims in published maps and institutional affiliations. 